\title{
Conformal Regge theory at finite boost
}

\author{
Simon Caron-Huot and Joshua Sandor \\ Department of Physics, McGill University, \\ Montréal, Québec, Canada \\ E-mail: schuot@mcgill.ca, jsandor@stanford.edu
}

Abstract: The Operator Product Expansion is a useful tool to represent correlation functions. In this note we extend Conformal Regge theory to provide an exact OPE representation of Lorenzian four-point correlators in conformal field theory, valid even away from Regge limit. The representation extends convergence of the OPE by rewriting it as a double integral over continuous spins and dimensions, and features a novel "Regge block". We test the formula in the conformal fishnet theory, where exact results involving nontrivial Regge trajectories are available.

Keywords: Conformal Field Theory, Integrable Field Theories

ArXiv EPrint: 2008.11759 


\section{Contents}

1 Introduction 1

2 Preliminaries 3

2.1 Review of conformal Regge kinematics 3

2.2 Review of conformal fishnet theory 4

3 Conformal Regge theory with exact energy dependence $\quad 6$

3.1 Sommerfeld-Watson resummation in S-matrix theory 7

3.2 Analytic continuation to the Lorentzian regime and Regge block 10

$\begin{array}{lll}3.3 & \text { Sommerfeld-Watson transformation } & 13\end{array}$

3.4 Formula for double-discontinuity: recovering Lorentzian inversion $\quad 15$

4 The Lorentzian fishnet model at subleading powers $\quad 17$

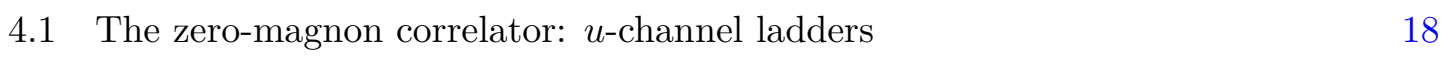

$4.2 t$-channel ladders and their double discontinuity 22

$\begin{array}{lll}4.3 & \text { The one-magnon correlator } & 23\end{array}$

$\begin{array}{llr}5 & \text { Conclusion } & 27\end{array}$

$\begin{array}{ll}\text { A Conformal blocks } & 27\end{array}$

B Harmonic polylogarithms and their analytic continuations 28

C Froissart-Gribov formula and cancellation of spurious poles $\quad 30$

D Lorentzian inversion of Regge block in $d=2$ and $d=4 \quad 31$

\section{Introduction}

The interactions between highly boosted objects is a topic of longstanding interest in relativistic field theory. On the on hand, due to time dilation effects, the Regge limit (large boost with fixed impact parameter) provides an instantaneous snapshot of essentially frozen objects. On the other hand, since probes move near the lightcone, observables in this limit are intrinsically dynamical and are strongly constrained by relativistic causality.

Many systems in the Regge limit exhibit a transient regime where interactions grow as a function of boost, before saturating as required by quantum mechanical conservation of probability. Regge theory quantifies the growth by the spin of effective excitations. A famous example is the rising hadronic cross-sections attributed to so-called Pomeron exchanges. Regge theory applies as well to highly boosted correlators in conformal field 
theories [1-3]. In strongly coupled, holographic CFTs, the dominant effective excitation is nothing but the bulk graviton. Its exchange grows as fast as allowed by the bound on chaos [4], making the consistency constraints mentioned above particularly stringent. Indeed, the fact that gravity grows with boost restricts its very structure at all energies [5]; more generally, growing amplitudes must satisfy positivity properties related to the Average Null Energy Condition [6].

In many studies of the Regge limit, it is often sufficient to consider only the leading term at large boost (in the intermediate growth regime). However, there may be situations where subleading effects are important. A simple example would be to study effects from photons in addition to gravitons. Another example would be saturation. Finally there may be theories where interactions do not grow, warranting precision studies. It was recently argued that the critical three-dimensional $\mathrm{O}(\mathrm{N})$ and Ising models are of this type, with Regge intercept less than unity $\left(j_{*}<1\right)$. This leads to transparent scattering at large boost $[7,8]$. In general, the Regge limit in conformal theories probes intermediate operators of large scaling dimension [9-11]. In transparent theories one might thus hope to use Regge theory, with exchange of a few dominant trajectories, to precisely bound the heavy spectrum, which could improve convergence of bootstrap calculations.

The goal of this paper is to extend formulas from Conformal Regge Theory so as to retain the exact energy dependence of four-point correlators.

Generally, the OPE in a conformal field theory converges whenever two local operators act on the vacuum, no matter where they are inserted in spacetime (see [12] for a review). The physical picture of effective Reggeized particles however arises from the OPE between an initial and a final state of a scattering process - often called the " $t$-channel" — and the OPE in such channels diverges. As reviewed below, this divergence occurs after Euclidean correlation functions are analytically continued to a "Regge sheet". Our main result is an exact resummation of the OPE which converges on the Regge sheet. Since correlators on the Euclidean sheet are well understood, we can state the result in terms of a difference or discontinuity:

$$
\begin{aligned}
\operatorname{Disc}_{14} \mathcal{G}(z, \bar{z})= & \int_{-\frac{d-2}{2}-i \infty}^{-\frac{d-2}{2}+i \infty} \frac{d J}{2 \pi i} \int_{\frac{d}{2}-i \infty}^{\frac{d}{2}+i \infty} \frac{d \Delta}{2 \pi i} \frac{e^{i \pi J} c^{t}(\Delta, J)+c^{u}(\Delta, J)}{\sin (\pi J)} \frac{R_{\Delta, J}^{(a, b)}(z, \bar{z})}{2 \kappa_{\Delta+J}^{(a, b)}} \\
& + \text { (subtractions) }
\end{aligned}
$$

The salient feature, familiar from (conformal) Regge theory $[2,3]$, is that a discrete sum over spins has been replaced by an integral. The power of Regge's idea is that this enlarges the radius of convergence of the OPE. We expect eq. (1.1) to converge anywhere on the Regge sheet.

The novel feature of eq. (1.1), in comparison with earlier work, is the "Regge block" $R_{\Delta, J}^{(a, b)}(z, \bar{z})$, defined in eq. (3.17) below, which accounts for subleading power corrections. Perhaps surprisingly, the Regge block is not simply the conformal block that one might have guessed from the leading-power formulas. The Regge block can be defined as the unique solution to conformal Casimir equations with a certain vanishing discontinuity. This combination turns out to cancel certain spurious poles, and we find that it neatly packages terms which otherwise might have been split in other treatments. 
Starting from eq. (1.1), concrete formulas for order-by-order asymptotic expansions in a given model can be obtained, as detailed in eq. (3.26).

These formulas will be tested in the conformal fishnet model [13], a recently proposed limit of $\mathcal{N}=4$ SYM that retains only scalar fields, but remains integrable at the cost of sacrificing unitarity. The OPE data corresponding to certain four-point correlators is known exactly and the correlators can be expanded in the coupling in terms of known special functions (harmonic polylogarithms). Using this expansion, we analytically continue correlators to the Lorentzian regime and compare their high-energy behaviour with eq. (1.1).

This paper is organized as follows. In section 2 we review kinematics of the Regge limit and the required analytic continuation, and we review the fishnet model. Section 3 derives our exact formula for the Regge limit, after reviewing analogous manipulations in the S-matrix context. We also obtain a formula for the double-discontinuity and confirm that it inverts the "Lorentzian inversion formula". In section 4 we test these formulas for various correlators in the fishnet model. Section 5 presents our brief conclusions.

\section{Preliminaries}

\subsection{Review of conformal Regge kinematics}

A conformal four-point correlator in Minkowski space $\mathcal{M}^{d-1,1}$ can be expressed as

$$
\left\langle\mathcal{O}_{4}\left(x_{4}\right) \mathcal{O}_{3}\left(x_{3}\right) \mathcal{O}_{2}\left(x_{2}\right) \mathcal{O}_{1}\left(x_{1}\right)\right\rangle=\frac{1}{\left(x_{12}^{2}\right)^{\frac{1}{2}\left(\Delta_{1}+\Delta_{2}\right)}\left(x_{34}^{2}\right)^{\frac{1}{2}\left(\Delta_{3}+\Delta_{4}\right)}}\left(\frac{x_{14}^{2}}{x_{24}^{2}}\right)^{a}\left(\frac{x_{14}^{2}}{x_{13}^{2}}\right)^{b} \mathcal{G}(z, \bar{z}),
$$

where $a=\frac{1}{2}\left(\Delta_{2}-\Delta_{1}\right), b=\frac{1}{2}\left(\Delta_{3}-\Delta_{4}\right)$ are combinations of the operators' scaling dimensions and the conformal cross-ratios $z, \bar{z}$ are related to the coordinates $\left\{x_{i}\right\}$ by

$$
z \bar{z}=\frac{\left(x_{1}-x_{2}\right)^{2}\left(x_{3}-x_{4}\right)^{2}}{\left(x_{1}-x_{3}\right)^{2}\left(x_{2}-x_{4}\right)^{2}}, \quad(1-z)(1-\bar{z})=\frac{\left(x_{1}-x_{4}\right)^{2}\left(x_{2}-x_{3}\right)^{2}}{\left(x_{1}-x_{3}\right)^{2}\left(x_{2}-x_{4}\right)^{2}} .
$$

The Regge limit of the correlation function is attained by applying large and opposite boosts to the pairs (12) and (34), sendind the operators to infinity along the lightcone:

$$
x_{1}^{+}, x_{3}^{-} \rightarrow-\infty, \quad x_{2}^{+}, x_{4}^{-} \rightarrow+\infty .
$$

Here we have rewritten the vectors in lightcone coordinates $x_{i}=\left(x_{i}^{+}, x_{i}^{-}, x_{i}^{a}, x_{i}^{b}\right)$. In the kinematics considered in this paper, both separations $\left(x_{4}-x_{1}\right)$ and $\left(x_{2}-x_{3}\right)$ are timelike while all other separations remain spacelike. To evaluate the Regge limit, the Lorentzian correlator must be obtained from the Euclidean theory described above. It is calculated by analytically continuing the theory from the region where $\bar{z}=z^{*}$, namely by rotating $\bar{z}$ around the branch point at $\bar{z}=1$ while keeping $z$ fixed [3]. The scattering process and analytic continuation are illustrated in figure 1.

To understand the continuation path a little more explicitly, we recall that for Lorentzian correlators, time-like distances acquire a small imaginary part $x_{23}^{2} \mapsto-\left|x_{23}\right|^{2} \pm i 0$ which is positive if the operators are in time-ordering and negative otherwise. The second cross-ratio in eq. (2.2) thus accumulates a phase $e^{2 \pi i}$ which is indeed what happens along the path. 

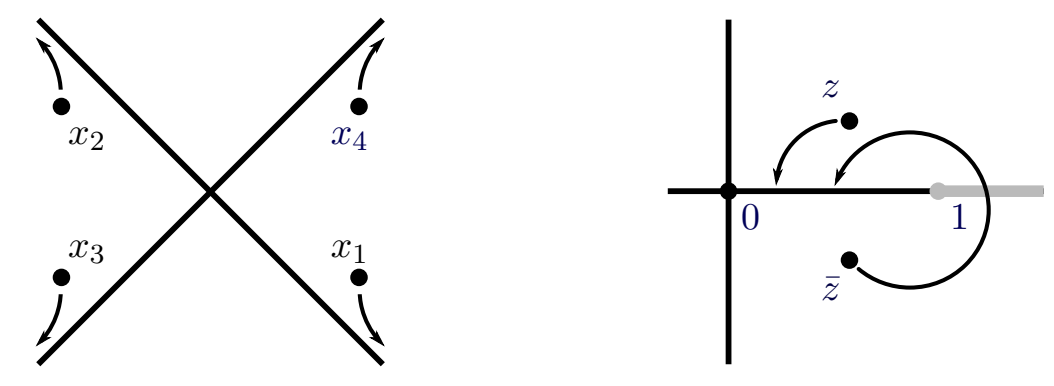

Figure 1. Four-point kinematics in the CFT Regge regime. Panel (a) illustrates the kinematics, in which time runs vertically, and panel (b) illustrates the analytic continuation $\mathcal{G}\left(z, \bar{z}^{\circlearrowleft}\right)$ : counterclockwise about the branch point. In the Euclidean regime $z^{*}=\bar{z}$ but both $z$ and $\bar{z}$ are real and independent in Lorentzian kinematics.

By further defining $\sigma^{2}=z \bar{z}$ and $w^{2}=z / \bar{z}$, we see that the Regge limit corresponds to $\sigma \rightarrow 0$ while $w$ is fixed. In analogy to the QFT Regge limit, we have an identification to the Mandelstam variables $\sigma \sim 1 / s$ and $w \sim t$ in $s$-channel scattering.

In our chosen kinematics (with pairs $(1,4)$ and $(2,3)$ in separate Rindler wedges) we have access to four operator orderings. Two are equivalent, and rather trivial: if one pair is time-ordered and the other anti-time-ordered, the continuation phases cancel out and the path does not leave the Euclidean sheet. All novel Lorentzian information is contained in commutators, or discontinuities, of which we can define two natural ones:

$$
\begin{aligned}
& \operatorname{Disc}_{23} \mathcal{G}(z, \bar{z}) \equiv-i e^{i \pi(a+b)}\left(\mathcal{G}\left(z, \bar{z}^{\circlearrowleft}\right)-\mathcal{G}(z, \bar{z})\right) \\
& \operatorname{Disc}_{14} \mathcal{G}(z, \bar{z}) \equiv-i\left(e^{i \pi(a+b)} \mathcal{G}\left(z, \bar{z}^{\circlearrowleft}\right)-e^{-i \pi(a+b)} \mathcal{G}(z, \bar{z})\right) .
\end{aligned}
$$

The different phases originate from the prefactor in eq. (2.1). These two discontinuities contain effectively the same information, and the fourth independent operator ordering, $\mathcal{G}\left(z, \bar{z}^{\circlearrowright}\right)$, can be reached by complex conjugation.

\subsection{Review of conformal fishnet theory}

Conformal fishnet theory is a recently proposed integrable theory in $d=4$ that is neither a gauge theory nor supersymmetric [13]. A chief interest of this theory is the fact that very few Feynman diagrams contribute to any given process - often a unique diagram at each loop order (or at each order in the 't Hooft $1 / N$ expansion). In this way, integrability of the theory allows for the calculation of certain Feynman diagrams which have been incalculable thus far by standard methods.

The theory contains two complex (matrix-valued) scalar fields, and its simplification comes at the price of unitarity: the basic 4-point interaction includes a term $\operatorname{Tr}\left(Y^{\dagger} X^{\dagger} Y X\right)$ but not its complex conjugate. Non-unitarity means that certain formulas below will contain unusual factors of the imaginary number $i$, but there otherwise appears to be no obstructions to resumming perturbation theory and discuss finite-coupling correlators.

An example of a class of diagrams which have been resummed to all orders in the planar limit are the "fishnet" diagrams drawn in figure 2. They describe the "zero-magnon" 


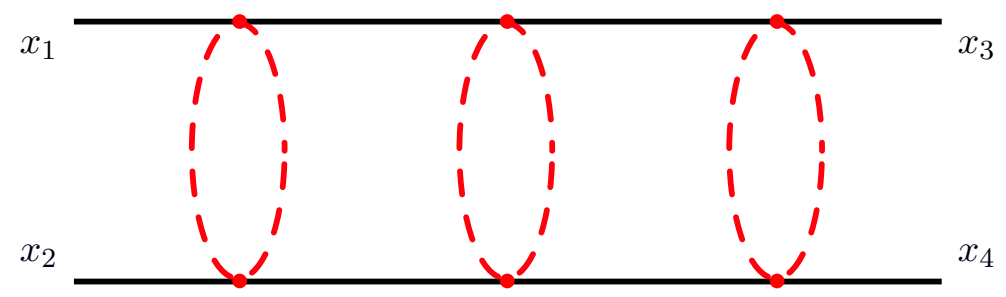

Figure 2. An example of zero-magnon $u$-channel fishnet ladder diagram evaluated in the computation of $\mathcal{G}^{u}(z, \bar{z})$. The dashed/solid four-point interaction sites have coupling $\xi^{2}$ so this diagram contributes at order $\xi^{12}$. The only other diagrams are of this form but with any number of "rungs".

correlator (trace implied):

$$
\left\langle 0\left|X^{\dagger}\left(x_{4}\right) X^{\dagger}\left(x_{3}\right) X\left(x_{2}\right) X\left(x_{1}\right)\right| 0\right\rangle \equiv \frac{1}{x_{12}^{2} x_{34}^{2}} \mathcal{G}(z, \bar{z})
$$

which was computed exactly in the $u$-channel in [14] (eqs. (3.12) and (A.6) there) as a sum over conformal blocks

$$
\mathcal{G}^{u}(z, \bar{z})=\sum_{J \geq 0}(-1)^{J} \int_{-\infty}^{\infty} \frac{d \nu}{2 \pi} C_{\Delta, J} \frac{256 E_{\Delta, J}}{1-256 \xi^{4} E_{\Delta, J}} G_{\Delta, J}^{(0,0)}(z, \bar{z}),
$$

where the scaling dimension is $\Delta=2+i \nu$,

$$
E_{\Delta, J}=\frac{1}{16(-\Delta+J+2)(-\Delta+J+4)(\Delta+J-2)(\Delta+J)}
$$

and the normalization coefficient is $[14]^{1}$

$$
C_{\Delta, J}=\frac{\Gamma(\Delta-1) \Gamma(2+J) \Gamma\left(\frac{1}{2}(\Delta+J)\right)^{2} \Gamma(4-\Delta+J)}{2 \Gamma(J+1) \Gamma(\Delta-2) \Gamma(\Delta+J-1) \Gamma\left(2-\frac{1}{2}(\Delta-J)\right)^{2}} .
$$

The conformal block $G_{\Delta, J}^{(a, b)}(z, \bar{z})$ is a combination of hypergeometric functions in $d=4$, see appendix A. For this calculation, we have $\Delta_{1}=\Delta_{2}=\Delta_{3}=\Delta_{4}=1$ and hence $a=b=0$. The $t$-channel ladders are given by the same expression $(2.6)$ without the $(-1)^{J}$. We also require $\xi^{2}$ to have a small and negative imaginary component for the $\nu$ integration to well-defined [14].

Eq. (2.6) can be related to the usual operator product expansion by noticing that since the conformal blocks decay exponentially as $\operatorname{Im}(\nu) \rightarrow-\infty$, we can close the integration contour in the lower half-plane and apply Cauchy's residue theorem. The poles of the integrand occur when $\nu$ solves

$$
1-256 \xi^{4} E_{2+i \nu, J}=0
$$

while all other poles are spurious and cancel in pairs [14]. The spectrum of this correlator thus consists of exactly two Regge trajectories: only two operators contribute for each spin.

\footnotetext{
${ }^{1}$ We removed an overall factor of $1 /(4 \pi)^{2}$ in eq. (2.5) and absorbed factors of 2 and $\pi$ into $C$.
} 
This result is valid for any finite coupling $\xi$, and in particular to all orders in perturbation theory, where the correlator is expanded as

$$
\mathcal{G}^{u}(z, \bar{z})=\frac{z \bar{z}}{z-\bar{z}} \sum_{n=0}^{\infty}\left(i \xi^{2}\right)^{n} \mathcal{G}^{(n)}(z, \bar{z}) .
$$

By analyzing the series expansions in small $z$ and $\bar{z}$, the authors of [14] found that $\mathcal{G}^{(n)}$ 's are combinations of single-valued harmonic polylogarithms (HPLs), a basis for special iterated integrals. Several useful properties and definitions of these functions are reviewed in appendix B. For example,

$$
\mathcal{G}^{(0)}(z, \bar{z})=z-\bar{z}
$$

and the order $\xi^{2}$ contribution is

$$
\begin{aligned}
\mathcal{G}^{(1)}(z, \bar{z})=\mathcal{L}_{1,0}(z, \bar{z})-\mathcal{L}_{1,0}(z, \bar{z}) \equiv & H_{1,0}(z)-H_{1,0}(\bar{z})+H_{1}(z) H_{0}(\bar{z}) \\
& -H_{0}(z) H_{1}(\bar{z})+H_{0,1}(\bar{z})-H_{0,1}(z) .
\end{aligned}
$$

This particular function can be written explicitly in terms of ordinary dilogarithms (see eq. (B.4)):

$$
\mathcal{G}^{(1)}(z, \bar{z})=-2 \operatorname{Li}_{2}(z)+2 \operatorname{Li}_{2}(\bar{z})+(\log (1-\bar{z})-\log (1-z)) \log (z \bar{z}),
$$

where $\mathrm{Li}_{2}$ is the dilogarithm function. We verified the formulas provided in ref. [14] up to 6-loops (order $\xi^{12}$ ) and order $\sigma^{4}$.

The functions $\mathcal{G}^{(L)}$ provide a "data mine" on which we can precisely test conformal Regge theory. Regge theory allows us to resum the OPE beyond its radius of convergence in cross-ratio space and to evaluate the correlator in the Regge limit via eq. (1.1). Our first goal will be to check that this agrees, order by order in the coupling and power by power in $\sigma$, with the analytic continuation of the $\mathcal{G}^{(L)}$ 's. A schematic of the calculation is provided in figure 3. We also applied this technique to the "one-magnon" four-point function, which has very similar structure to the zero-magnon case and is reviewed in section 4.3. The Regge limit at leading power has been preceedingly studied in ref. [15] and was extended to other fishnet correlators in $[16,17]$.

\section{Conformal Regge theory with exact energy dependence}

The extension of the $s$-channel OPE to the Regge limit described in section 2.1 was obtained in the seminal paper [3]. This is nontrivial since the sum over spins diverges in the Regge regime. The solution is to rewrite the sum as an integral via the so-called SommerfeldWatson transform. Our contribution here will be to extend the formulas of [3] to an exact expression (see eqs. (3.24)-(3.26) below) which can be used to obtain arbitrary subleading powers of $z, \bar{z}$. As we will see, a new sort of term then appears. In this section we keep the spacetime dimension and external operator dimensions generic. 


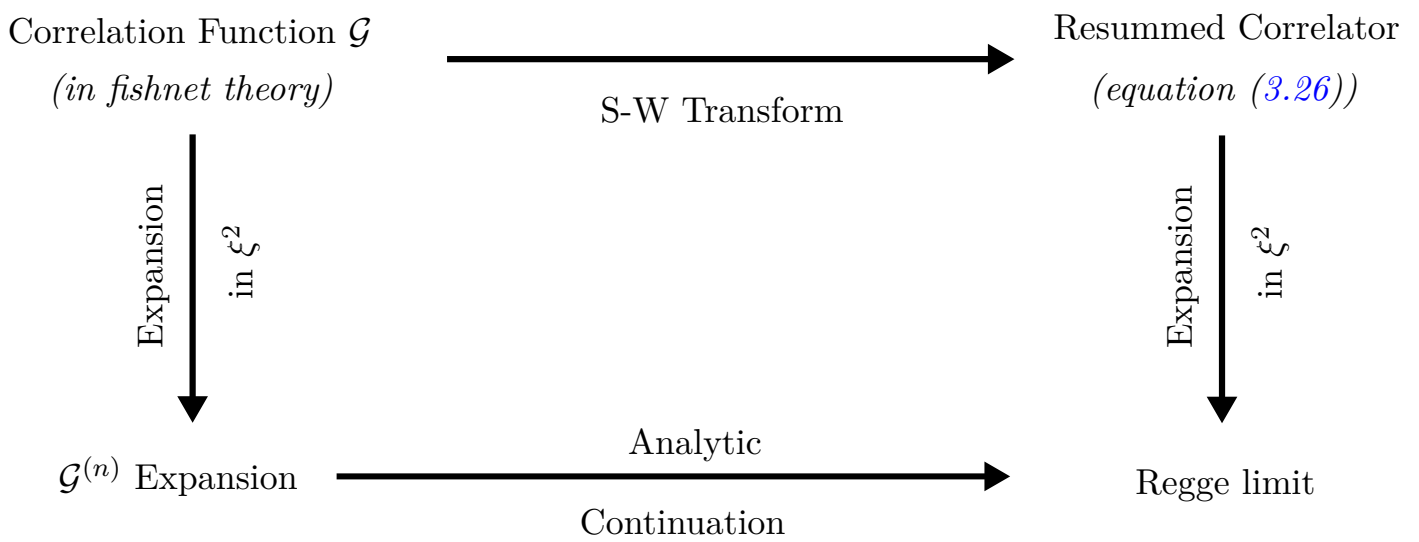

Figure 3. A sketch of the processes evaluated in the following sections. We analyze the SommerfeldWatson resummation in generic conformal theories and demonstrate that the diagram commutes in the fishnet model.

\subsection{Sommerfeld-Watson resummation in S-matrix theory}

We begin by reviewing the classic resummation of $\mathrm{SO}(d)$ spherical harmonics, which will give us intuition about what we should, and should not, expect (see also [18, 19]). Consider a function of one angle:

$$
F(\cos \theta)=\sum_{J} a_{J} C_{J}(\cos \theta)
$$

where the $\mathrm{SO}(d)$ spherical harmonics $C_{J}$ are defined in eq. (A.5). We use a normalization which trivializes the Regge $\operatorname{limit} \lim _{x \rightarrow \infty} C_{J}(x) \rightarrow(2 x)^{J}$. We will borrow nomenclature from S-matrix theory, where in $d$ spacetime dimensions one would use $\mathrm{SO}(d-1)$ partial waves, $\cos \theta=1+\frac{2 t}{s}$ (say for massless scattering), and the coefficients would depend on center-of-mass energy $s$. Regge theory aims to use such $s$-channel partial waves to study the large- $t$, fixed- $s$ Regge limit; the $s$-dependence will play no role in our discussion.

As reviewed below, in S-matrix applications the partial waves $a_{J}$ are the sum of a part which is analytic and one which alternates with spin:

$$
a_{J}=a_{J}^{t}+(-1)^{J} a_{J}^{u}
$$

where each of $a_{J}^{t, u}$ is analytic and polynomially bounded in a half-plane $\operatorname{Re}(J)>j_{*}$. These are associated with $t$ - and $u$-channel cuts, representing singularities at positive and negative $x$, respectively. Many references use instead even- and odd- combinations $\left(a_{J}^{t} \pm a_{J}^{u}\right)$.

To rewrite the sum (3.1) as an integral, we need to think of the analytic properties of the spherical harmonics $C_{J}(x)$. These are entire functions of $J$ (except for the gamma function poles at negative $J$ ) which generally have a " $u$-channel" cut for $x \in(\infty,-1]$. In fact we have two natural functions: $C_{J}( \pm x)$. They are related by an overall sign $(-1)^{J}$ when the spin is an integer but generally they are distinct. The Sommerfeld-Watson transform pairs $a_{J}^{t}$ with the function with a $t$-cut, and $a_{J}^{u}$ with the function with a $u$-cut:

$$
F(x)=-\pi \int_{C} \frac{d J}{2 \pi i} \frac{a_{J}^{t} C_{J}(-x)+a_{J}^{u} C_{J}(x)}{\sin (\pi J)}+(\text { subtractions })
$$



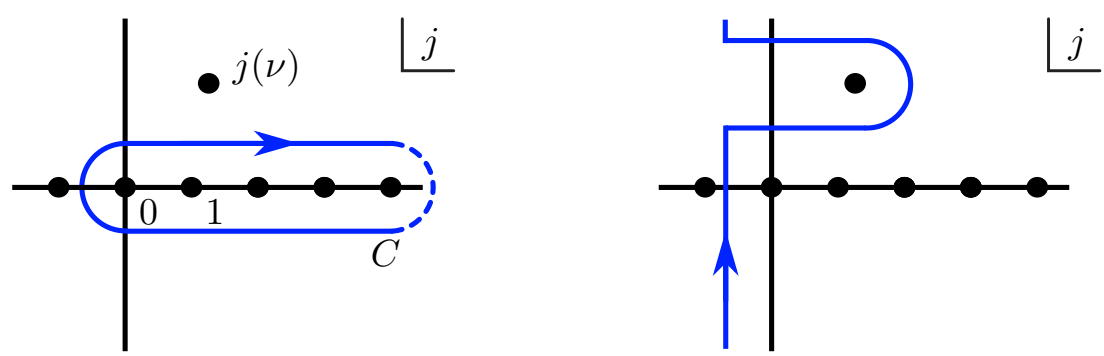

Figure 4. First step of the Sommerfeld-Watson transform in S-matrix theory. The contour should remain to the right of $j$-plane singularities. The contours are equivalent in Euclidean kinematics but the second one allows a safe continuation to Lorentzian.

where the contour $C$ encircles clockwise the poles of $1 / \sin (\pi J)$ with $J \geq 0$, see figure 4 . Since the residue of $1 / \sin (\pi J)$ is proportional to $(-1)^{J}$, it is easy to verify that the integral reproduces the sum in eq. (3.1). The subtractions are a polynomial in $x$, accounting for the possibility that for a finite number of spins the analytic continuation of $a_{J}^{t}$ may not agree with the coefficient in eq. (3.1).

On the contour $C$, the integral (3.3) converges when the original sum does, ie. when $|\cos \theta|$ is not too large. To gain anything from this trick one must deform the contour to a vertical line. It will be convenient to center it on the fixed line of the Weyl reflection $J \mapsto 2-d-J:$

$$
F(x)=-\pi \int_{-\frac{d-2}{2}-i \infty}^{-\frac{d-2}{2}+i \infty} \frac{d J}{2 \pi i} \frac{a_{J}^{t} C_{J}(-x)+a_{J}^{u} C_{J}(x)}{\sin (\pi J)}+\text { (subtractions). }
$$

The contour should remain to the right of all singularities of the $a_{J}^{t, u}$. In Euclidean kinematics, $C_{J}(\cos \theta) \sim e^{ \pm i \theta J}$ at large imaginary $J$ and the integral converges (possibly as a distribution) as long as $\theta \in[0, \pi]$. In Lorentzian kinematics with $|x| \gg 1, C_{J}(x) \sim(2 x)^{J}$ and we retain convergence on this contour as long as $\arg (x) \in[0, \pi]$. We stress that, given $a_{J}^{t}$ and $a_{J}^{u}$, eq. (3.4) is an exact representation for the function $F(x)$.

Let us comment on the meaning of the coefficients $a_{J}^{t, u}$ in eq. (3.4). In general, they are analytic functions in some half-plane $\operatorname{Re}(J) \geq j_{*}$ which may not include the vertical line $\operatorname{Re}(J)=-\frac{d-2}{2}$. In drawing the second contour in figure 4 we assumed that singularities occur at finite $\operatorname{Im} J$, so that there are no obstructions to reaching the vertical line at large imaginary $J$. This seems physically reasonable since large-spin is often a semi-classical limit. The same comment will apply below in CFT.

A typical application of eq. (3.4) is to obtain large- $x$ asymptotics. The intermediate steps are subtle if one is interested in subleading terms, but since the answer is surprisingly simple, it will be worth going through the steps (following appendix A of [19]). The basic idea is to split $C_{J}(x)$ into two parts, which decay in the left and right $J$ half-planes respectively:

$$
C_{J}(x)=C_{J}^{\text {pure }}(x)+\frac{\Gamma(J+d-2) \Gamma\left(-\frac{d-2}{2}-J\right)}{\Gamma\left(J+\frac{d-2}{2}\right) \Gamma(-J)} C_{2-d-j}^{\text {pure }}(x),
$$




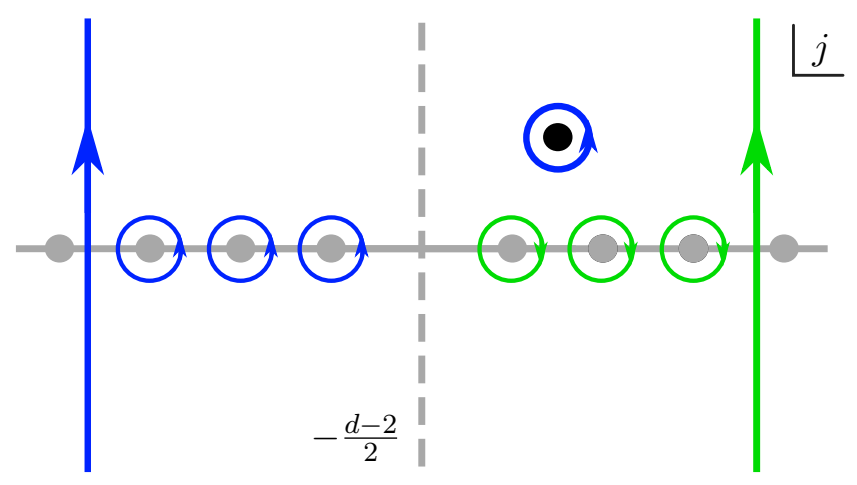

Figure 5. Spurious poles of type 2 and 3 cancel each other. The physical Regge pole is shaded black.

where $C_{J}^{\text {pure }}(x)$ satisfies the same Casimir equation but contains a single tower of term as $x \rightarrow \infty$ (this function is proportional to Legendre's $Q$ when $d=3$ ):

$$
C_{J}^{\text {pure }}(x) \equiv(2 x)^{J}{ }_{2} F_{1}\left(-\frac{J}{2}, \frac{1-J}{2}, 2-\frac{d}{2}-J, \frac{1}{x^{2}}\right) .
$$

We should thus deform the integration contour left for the $C_{J}^{\text {pure }}$ terms, and right for $C_{2-d-J}^{\text {pure }}$. In principle, one has to include the following types of singularities:

1. Physical left poles or cuts from $a_{j}^{t, u}$.

2. Spurious left poles at $J=-\frac{d-2}{2}-m, m=1,2,3 \ldots$ from $a_{J}^{t, u}$ (see below).

3. Right poles at $J=-\frac{d-2}{2}+m, m=1,2,3 \ldots$ from gammas in eq. (3.5).

4. Left poles at $J=-1,-2 \ldots$ from $1 / \sin (\pi J)$.

5. Right poles at $J=0,1,2 \ldots$ from $1 / \sin (\pi J)$.

The surprise, remarkably, is that poles 2-5 all cancel out, and only the physical singularities of $a_{j}^{t, u}$ contribute! In brief, poles 2-3 are related by a Weyl reflection and cancel in pairs; poles 4 are generically absent due to a cancellation between $t$ - and $u$-channel coefficients, ${ }^{2}$ and poles 5 are absent due to $1 / \Gamma(-J)$ in eq. (3.5). The cancellations are detailed in appendix $\mathrm{C}$ and can be readily understood using a concrete formula for the $a_{J}^{t, u}$, known as the Froissart-Gribov formula.

The upshot is that only physical singularities (as defined precisely in the appendix) contribute. Considering, for notational simplicity, the case in which these consist of discrete poles at $J=j_{n}$, and taking $x$ to be positive and above the real axis cut, the result is:

$$
\lim _{x \rightarrow \infty} F(x+i 0)=-\pi \sum_{j_{n}} \operatorname{Res}_{J=j_{n}} \frac{e^{-i \pi J} a_{J}^{t}+a_{J}^{u}}{\sin (\pi J)} C_{J}^{\text {pure }}(x) .
$$

\footnotetext{
${ }^{2}$ By "generically" we mean that the combination $\frac{e^{-i \pi J} a_{J}^{t}+a_{J}^{u}}{\sin (\pi J)}$ has a pole at $J=-m$ with $m=$ integer if and only if the theory has a physical Regge pole at that location, corresponding to an integer-power term in the Regge limit, which is not the case in a generic theory.
} 
This is a fundamental result of Regge theory. Since $C_{J}^{\text {pure }}(x) \approx(2 x)^{J} \propto t^{J}$, the rightmost $J$-plane singularities dominate at $t \gg s$. More generally, the sum gives an asymptotic expansion in $1 / t$. The phases of the two terms in eq. (3.7) are simply those of $(-t-i 0)^{J}$ and $(-u-i 0)^{J}$, respectively.

The remarkable feature of eq. (3.7) is that, to correctly reproduce the amplitude to any desired order in the $1 / t$ expansion of $F$, it suffices to replace $C_{J}$ by $C_{J}^{\text {pure }}$ in the Sommerfeld-Watson formula (3.4) and ignore spurious singularities of $1 / \sin (\pi J)$ and $a_{J}^{t, u}$.

\subsection{Analytic continuation to the Lorentzian regime and Regge block}

The spectral representation of correlation functions is the starting point for Regge analysis in conformal theories. It allows to write the correlation function as an integral over continuous dimensions:

$$
\mathcal{G}(z, \bar{z})=\sum_{J} \int_{-\infty}^{\infty} \frac{d \nu}{2 \pi} c(\Delta, J) F_{\Delta, J}^{(a, b)}(z, \bar{z})+\text { (non-norm) }
$$

where the exchanged operator scaling dimension is parametrized as $\Delta=d / 2+i \nu$, where $\nu$ is a complex number. The meromorphic function $c(J, \Delta)$ contains the OPE coefficient data of a particular theory, and has poles at the location of local operators. The "nonnormalizable" modes account for operators with $\Delta<d / 2$ (which includes, notably, the identity) [20]. The conformal partial waves $F_{J, \Delta}$ are a sum of conformal block and its shadow [3, 21, 22],

$$
F_{\Delta, J}^{(a, b)}(z, \bar{z})=\frac{1}{2}\left(G_{\Delta, J}^{(a, b)}(z, \bar{z})+\frac{K_{d-\Delta, J}^{(a, b)}}{K_{\Delta, J}^{(a, b)}} G_{d-\Delta, J}^{(a, b)}(z, \bar{z})\right),
$$

with coefficient that are products of gamma functions

$$
K_{\Delta, J}^{(a, b)}=\frac{\Gamma(\Delta-1)}{\Gamma\left(\Delta-\frac{d}{2}\right)} \kappa_{\Delta+J}^{(a, b)}, \quad \kappa_{\beta}^{(a, b)}=\frac{\Gamma\left(\frac{\beta}{2}-a\right) \Gamma\left(\frac{\beta}{2}+a\right) \Gamma\left(\frac{\beta}{2}-b\right) \Gamma\left(\frac{\beta}{2}+b\right)}{2 \pi^{2} \Gamma(\beta-1) \Gamma(\beta)} .
$$

The spectral representation (3.8) involves a discrete sum over spins, analogous to eq. (3.1). To reach the Lorentzian regime, we must first replace the sum by an integral, and then analytically continue $\bar{z}$ counterclockwise around 1 .

This process has been discussed many times, but we found an unexpected twist: the first step enjoys some freedom because one can add to $F_{\Delta, J}$ terms which vanish for integer spin. We find that the next steps is greatly simplified, especially at subleading powers, by making such an improvement. This discussion will be somewhat technical.

Let us recall the defining properties of $F_{\Delta, J}$ : it satisfies the same Casimir equation as $G_{\Delta, J}$, and it is Euclidean single-valued (meaning, it has no branch cut when $\bar{z}=z^{*}$ ). The problem with eq. (3.9) is that this property does not hold for non-integer spin - this combination is then not natural in any sense! In fact no combination of $G$ 's can satisfy Euclidean single-valuedness for non-integer $J$, because it is violated in the $z, \bar{z} \rightarrow 0$ limit:

$$
\lim _{z \ll \bar{z} \ll 1} G_{\Delta, J}^{(a, b)}=(z \bar{z})^{\Delta / 2}(z / \bar{z})^{J / 2} .
$$


The last factor is only Euclidean single-valued when $J$ is an integer. Our proposed resolution is that one can still impose Euclidean single-valuedness in either the left or right half-plane. Given our kinematics of interest, we pick the second option, meaning in particular that we cancel the monodromy around the point $(z, \bar{z})=(1,1)$. To construct the corresponding block, we make an ansatz using the three functions: $G_{\Delta, J}, G_{d-\Delta, J}$ and $G_{J+d-1,1-\Delta}$. These solve the same Casimir equation, are regular at $z=\bar{z}$, and do not have contain singular powers $(z \bar{z})^{-J / 2}$ at positive $J$. Using the method detailed shortly, we find that the natural non-integer spin version of eq. (3.9) contains a third term:

$$
\begin{aligned}
F_{\Delta, J}^{(a, b) \operatorname{good}}(z, \bar{z})= & \frac{1}{2} G_{\Delta, J}^{(a, b)}(z, \bar{z})+\frac{1}{2} \frac{K_{d-\Delta, J}}{K_{\Delta, J}} G_{d-\Delta, J}^{(a, b)}(z, \bar{z})+ \\
& +\pi K_{J+d-1,1-\Delta} \frac{\Gamma\left(-J-\frac{d-2}{2}\right)}{\Gamma(-J)}\left(s_{\Delta+J}^{(a, b)}-s_{\Delta+2-d-J}^{(a, b)}\right) G_{J+d-1,1-\Delta}^{(a, b)}(z, \bar{z}),
\end{aligned}
$$

where $s$ is a product of sines which will often reoccur:

$$
s_{\beta}^{(a, b)}=\frac{\sin \pi\left(\frac{\beta}{2}+a\right) \sin \pi\left(\frac{\beta}{2}+b\right)}{\sin (\pi \beta)} .
$$

For the moment, we remark only that the second line of eq. (3.12) manifestly vanishes for integer $J \geq 0$, due to $1 / \Gamma(-J)$, so $F^{\text {good }}$ reduces to $F$ in that case. Also, trigonometric identities can be used to show that the definition is invariant under the symmetry $(a, b) \mapsto$ $(-a,-b)$. To our knowledge, the function in eq. (3.12) is new. It would be interesting to interpret it in the language of shadow representation, light transforms or integrability [21, $23,24]$, and also to compare with the function called $\mathcal{G}$ in ref. [25].

Our method to analytically continue $F_{\Delta, J}^{(a, b) \operatorname{good}}(z, \bar{z})$ to the Regge sheet, following the path in figure 1, is the same method that we used to find the coefficients in eq. (3.12). We first decompose each block $G_{\Delta, J}^{(a, b)}$ into pure power solutions according to

$$
G_{\Delta, J}^{(a, b)}(z, \bar{z})=g_{\Delta, J}^{(a, b) \text { pure }}(z, \bar{z})+\frac{\Gamma(J+d-2) \Gamma\left(-J-\frac{d-2}{2}\right)}{\Gamma\left(J+\frac{d-2}{2}\right) \Gamma(-J)} g_{\Delta,-J-d+2}^{(a, b) \text { pure }}(z, \bar{z}),
$$

where each $g^{\text {pure }}$ contains a single tower of terms in the limit (3.11). This decomposition is identical to that used for spherical harmonics in eq. (3.5). Contrary to $G$, the $g^{\text {pure }}$ 's are not symmetrical in $(z, \bar{z})$. They are however easy to analytically continue around $\bar{z}=1$ : since $z$ is held fixed during the continuation, the exponent of $z$ cannot change $[3,11,22]$ :

$$
g_{\Delta, J}^{(a, b) \text { pure }}\left(z, \bar{z}^{\circlearrowleft}\right)=\left(1-2 i e^{-i \pi(a+b)} s_{\Delta+J}^{(a, b)}\right) g_{\Delta, J}^{(a, b) \text { pure }}(z, \bar{z})-\frac{i}{\pi} \frac{e^{-i \pi(a+b)}}{\kappa_{\Delta+J}^{(a, b)}} g_{1-\Delta, 1-J}^{(a, b) \text { pure }}(z, \bar{z}) .
$$

The continuation of $F_{\Delta, J}^{(a, b)}(z, \bar{z})$ or $F_{J, \Delta}^{(a, b) \operatorname{good}}(z, \bar{z})$ leaves us with eight $g^{\text {pure's with various }}$ complicated coefficients.

Now the crux is that a combination of blocks $F(z, \bar{z})$ is Euclidean single-valued around $(1,1)$ if and only if the $g^{\text {pure }} \mathrm{s}$ can be re-packaged into $G$ 's. The reason is that we can reach the Regge sheet by rotating $z, \bar{z}$ counter-clockwise starting from the region $z, \bar{z}>1$, where 


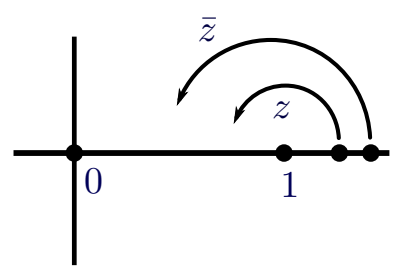

Figure 6. Rotation of $z, \bar{z}$ counterclockwise from $z, \bar{z}>1$.

Euclidean single-valued functions are symmetrical: $F(z+i 0, \bar{z}-i 0)=F(z-i 0, \bar{z}+i 0)$ (see figure 6 ). Since we can reach the Regge sheet by continuing $z, \bar{z}$ symmetrically from a region where the correlator is symmetrical, it follows that the continuation of a singlevalued correlator is also symmetrical: $F\left(z, \bar{z}^{\circlearrowleft}\right)=F\left(z^{\circlearrowleft}, \bar{z}\right)$ (and nonsingular at $z=\bar{z}$ ). This property ensures that it is a sum of $G$ 's. This property fails for non-integer spins for the combination $F_{\Delta, J}^{(a, b)}$, but it is restored by the unique combination $F_{\Delta, J}^{(a, b) \operatorname{good}}$. This is how we determined eq. (3.12).

The coefficients of the four resulting $G$ 's contain a part that is essentially the original $F_{\Delta, J}^{(a, b) \text { good }}$. We thus subtract those off and record the discontinuity:

$$
\begin{aligned}
\operatorname{Disc}_{14} F_{\Delta, J}^{(a, b) \operatorname{good}}(z, \bar{z}) & =-i\left(e^{i \pi(a+b)} F_{\Delta, J}^{(a, b) \operatorname{good}}\left(z, \bar{z}^{\circlearrowleft}\right)-e^{-i \pi(a+b)} F_{\Delta, J}^{(a, b) \operatorname{good}}(z, \bar{z})\right) \\
& \equiv-\frac{R_{\Delta, J}^{(a, b)}(z, \bar{z})}{2 \pi \kappa_{\Delta+J}^{(a, b)}},
\end{aligned}
$$

which is given in terms of a new "Regge block":

$$
\begin{aligned}
R_{\Delta, J}^{(a, b)}= & G_{1-J, 1-\Delta}^{(a, b)}-\kappa_{\Delta+J}^{\prime(a, b)} G_{\Delta, J}^{(a, b)}-\frac{\Gamma(d-\Delta-1) \Gamma\left(\Delta-\frac{d}{2}\right)}{\Gamma(\Delta-1) \Gamma\left(\frac{d}{2}-\Delta\right)} \kappa_{d-\Delta+J}^{\prime(a, b)} G_{d-\Delta, J}^{(a, b)} \\
& +\frac{\Gamma(J+d-2) \Gamma\left(-J-\frac{d-2}{2}\right)}{\Gamma\left(J+\frac{d-2}{2}\right) \Gamma(-J)} \kappa_{\Delta+J}^{\prime(a, b)} \kappa_{d-\Delta+J}^{\prime(a, b)} G_{J+d-1,1-\Delta}^{(a, b)} .
\end{aligned}
$$

Here we defined the following product of $\Gamma$-functions:

$$
\kappa_{\beta}^{\prime(a, b)}=\frac{r_{\beta}^{(a, b)}}{r_{2-\beta}^{(a, b)}} \quad \text { with } \quad r_{\beta}^{(a, b)} \equiv \frac{\Gamma\left(\frac{\beta}{2}+a\right) \Gamma\left(\frac{\beta}{2}+b\right)}{\Gamma(\beta)} .
$$

Importantly, the continuation (3.16) is exact even for noninteger $J$.

The first term of (3.17) dominates in the Regge limit, $G_{1-J, 1-\Delta}^{(a, b)} \sim \sigma^{1-J}$, and it (deservedly) receives the most attention $[3,11,23]$. However we will find that the other terms contribute nontrivially at subleading powers.

A simple defining property of $R_{\Delta, J}^{(a, b)}$ is that, being a discontinuity of blocks, its other discontinuity vanishes:

$$
\operatorname{Disc}_{23} R_{\Delta, J}^{(a, b)}(z, \bar{z})=0 .
$$

We find that eq. (3.17) is the only combination of $G$ 's satisfying this. Alternatively, one could have defined a Regge block by taking the other discontinuity, $R^{\prime} \propto \operatorname{Disc}_{23} F_{\Delta, J}^{(a, b) \text { good }}$ : this is given by the same expression (3.17) but with $\kappa_{\beta}^{\prime(-a,-b)}$. 

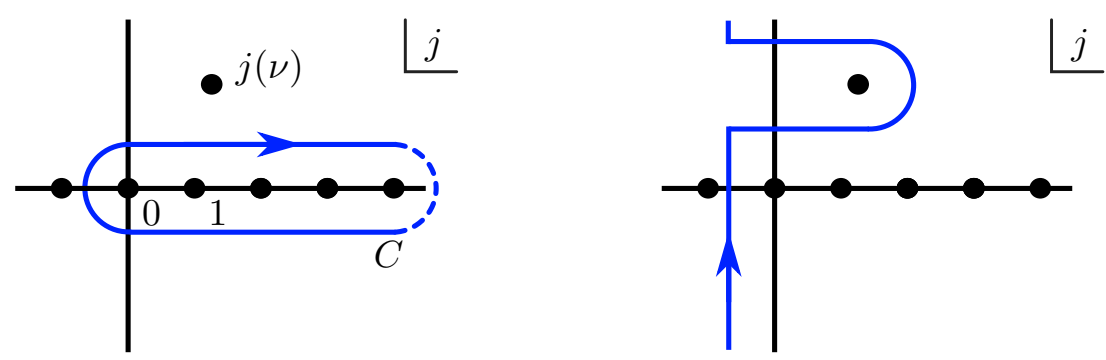

Figure 7. Similar to figure 4: the Sommerfeld-Watson transform for the $F_{\Delta, J}^{(a, b) \text { good }}(z, \bar{z})$ block. The contour should remain to the right of singularities of $c^{t, u}$. The contours are equivalent in Euclidean kinematics, and the second one allows a safe continuation to Lorentzian.

\subsection{Sommerfeld-Watson transformation}

With the analytic continuation of blocks worked out, one can try to evaluate the continued correlation function following the path figure 1 and discontinuity:

$$
\operatorname{Disc}_{14} \mathcal{G}(z, \bar{z}) \stackrel{?}{=} \sum_{J \geq 0}^{\infty} \int_{-\infty}^{\infty} \frac{d \nu}{2 \pi} c(\Delta, J) \frac{R_{\Delta, J}^{(a, b)}(z, \bar{z})}{2 \pi \kappa_{\Delta+J}^{(a, b)}} .
$$

After some inspection, one finds that this expression makes no sense: the Regge block $R_{\Delta, J}$ scales as $\sigma^{1-J}$ as $\sigma \rightarrow 0$ in the Regge limit, so the sum diverges.

Just as for the S-matrix Regge limit, the solution is to step back and rewrite the sum as an integral before analytically continuing the cross-ratios to take the discontinuity. This requires first promoting the spin $J$ to a complex variable and the partial wave coefficient $c(J, \Delta)$ to analytic functions of $J$. In the S-matrix case, this possibility was first observed by Regge and was soon proved generally by Froissart and Gribov; the analogous result in CFT was proved recently $[20,22,23]$. In general, this works for $J>j_{*}$ where it is known that $j_{*} \leq 1$ in a unitary theory.

The partial waves form not one, but in fact two analytic functions of spin:

$$
c(\Delta, J)=c^{t}(\Delta, J)+(-1)^{J} c^{u}(\Delta, J),
$$

where each term is nicely behaved (power-law bounded) at large imaginary $J$.

Regge's idea allows us to express the sum over integer spins as an integral in the complex plane,

$$
\mathcal{G}(z, \bar{z})=-\pi \int_{C} \frac{d J}{2 \pi i} \int_{-\infty}^{\infty} \frac{d \nu}{2 \pi} \frac{e^{i \pi J} c^{t}(\Delta, J)+c^{u}(\Delta, J)}{\sin (\pi J)} F_{\Delta, J}^{(a, b) \operatorname{good}}(z, \bar{z})
$$

where the contour $C$ envelopes the positive real $j$ axis, as illustrated in figure 7 . Once this contour is in place, we can drag it around the complex plane to obtain a form where analytic continuation is possible. The general technique is known as the SommerfeldWatson transform.

In the contour deformation of figure 7 we may encounter poles from the coefficients $c^{t, u}(\Delta, J)$, as well as possible spurious poles from $F$. Such spurious poles were discussed 


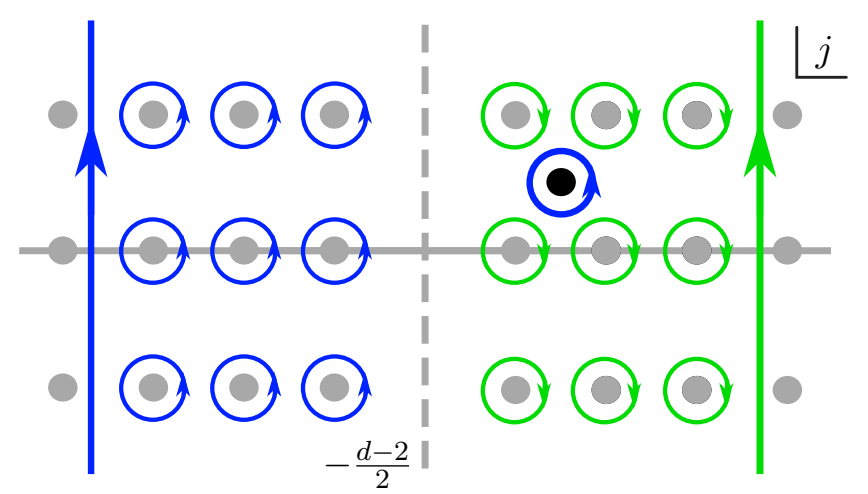

Figure 8. Spurious poles of type 2-3 cancel against each other just as in the S-matrix case (figure 5). Again, a physical Regge pole is shaded black.

in [2]. However, we find that these are absent when using the block $F^{\text {good }}$, for a simple reason: as the unique Casimir eigenfunction satisfying certain regularity conditions, $F_{\Delta, J}^{\text {good }}$ is automatically analytic for $\operatorname{Re}(J)>-\frac{d-2}{2}$ when $\Delta$ is along the principal series $\operatorname{Re}(\Delta)=\frac{d}{2}$. We have also verified explicitly the cancellation of poles using residue formulas from [26]. We can thus write eq. (3.22) with a vertical contour:

$$
\mathcal{G}(z, \bar{z})=-\pi \int_{-\frac{d-2}{2}-i \infty}^{-\frac{d-2}{2}+i \infty} \frac{d J}{2 \pi i} \int_{-\infty}^{\infty} \frac{d \nu}{2 \pi} \frac{e^{i \pi J} c^{t}(\Delta, J)+c^{u}(\Delta, J)}{\sin (\pi J)} F_{\Delta, J}^{(a, b) \operatorname{good}}(z, \bar{z}) .
$$

On this contour we are now allowed to analytically continue to the Regge sheet. In particular we can take the discontinuity directly under the integration sign to get the Regge block in eq. (3.17):

$$
\begin{aligned}
\operatorname{Disc}_{14} \mathcal{G}(z, \bar{z})= & \int_{-\frac{d-2}{2}-i \infty}^{-\frac{d-2}{2}+i \infty} \frac{d J}{2 \pi i} \int_{-\infty}^{\infty} \frac{d \nu}{2 \pi} \frac{e^{i \pi J} c^{t}(\Delta, J)+c^{u}(\Delta, J)}{\sin (\pi J)} \frac{R_{\Delta, J}^{(a, b)}(z, \bar{z})}{2 \kappa_{\Delta+J}^{(a, b)}} \\
& + \text { (subtractions) }
\end{aligned}
$$

We note that the sign of the phase $e^{i \pi J}$ is opposite in coordinate space as in momentum space. The sign is forced on us since during the continuation, the block contains a factor $F_{\Delta, J}^{(a, b) \operatorname{good}}(z, 1-i \epsilon) \sim e^{-\sqrt{i \epsilon} J}$ which grows at positive imaginary $J$. With the wrong choice $(-1)^{J} \mapsto e^{-i \pi J}$, the integral would diverge.

Eq. (3.24) is a central result of this paper: an exact representation of the correlator on the Regge sheet. This is a critical step toward obtaining asymptotic expansions, to which we now turn. The "subtractions" include the discontinuity of non-normalizable modes in eq. (3.8), and possible low-spin corrections as in eq. (3.3).

Similar to eq. (3.5), to derive an asymptotic expansion in the Regge limit $\sigma \rightarrow 0$ we simply write the Regge block as a term which decays on the left $J$-plane, plus the three remaining $G$ 's in eq. (3.17):

$$
R_{\Delta, J}^{(a, b)}(z, \bar{z})=G_{1-J, 1-\Delta}^{(a, b)}(z, \bar{z})+\text { rest. }
$$


We then deform the $J$-contour left on the first term, and right for the rest. Similar to section 3.1, we find the following types of poles:

1. Physical left poles or cuts from $c^{t, u}(\Delta, J)$.

2. Spurious left poles at $J=-\frac{d-2}{2}-m, J=1-\Delta-m$ and $J=1-\tilde{\Delta}-m, m=1,2,3 \ldots$ from $C^{t, u}(\Delta, J)$.

3. Right poles at $J=-\frac{d-2}{2}+m, J=1-\Delta+m$ and $J=1-\tilde{\Delta}+m$ from rest of eq. (3.17)

4. Left poles at $J=-1,-2 \ldots$ from $1 / \sin (\pi J)$.

5. Right poles at $J=0,1,2 \ldots$ from $1 / \sin (\pi J)$.

Poles of types 2-4 cancel by the same two mechanisms discussed above. Namely, types 2-3 cancel among Weyl-reflected pairs $J \mapsto 2-J-d$ (see figure 8), by the mechanism detailed in eq. (C.5). The crux is that argument is that the Regge block $R^{(a, b)}$ is free of spurious poles. Type 4 poles multiply an explicit zero in the Lorentzian inversion formula, and so are generically absent in the sense discussed in the S-matrix case. Poles of type 5 however do not cancel in the CFT case and must be retained. The result is the following asymptotic expansion in the Regge limit, including subleading powers:

$$
\begin{aligned}
\lim _{\sigma \rightarrow 0} \operatorname{Disc}_{14} \mathcal{G}(z, \bar{z})= & \int_{-\infty}^{\infty} \frac{d \nu}{2 \pi} \sum_{j_{n}(\Delta)} \operatorname{Res}_{J=j_{n}(\Delta)} \frac{e^{i \pi J} c^{t}(\Delta, J)+c^{u}(\Delta, J)}{\sin (\pi J)} \frac{G_{1-J, 1-\Delta}^{(a, b)}(z, \bar{z})}{2 \kappa_{\Delta+J}^{(a, b)}} \\
& -\sum_{J \geq 0} \int_{-\infty}^{\infty} \frac{d \nu}{2 \pi} c(\Delta, J) s_{\Delta+J}^{(-a,-b)} 2 F_{\Delta, J}^{\prime(a, b)}(z, \bar{z}) .
\end{aligned}
$$

The first line could have been easily guessed and is as in S-matrix Regge theory (see eq. (3.7)). The second line is a new contribution which to our knowledge has not been discussed explicitly before; it is important at subleading orders. The block $F^{\prime}$ is defined similarly to eq. (3.9) with $\kappa \mapsto \kappa^{\prime}$ from eq. (3.18):

$$
F_{\Delta, J}^{\prime(a, b)}(z, \bar{z})=\frac{1}{2}\left(G_{\Delta, J}^{(a, b)}(z, \bar{z})+\frac{\kappa_{d-\Delta+J}^{\prime(a, b)}}{\kappa_{\Delta+J}^{\prime(a, b)}} \frac{\Gamma(d-\Delta-1) \Gamma\left(\Delta-\frac{d}{2}\right)}{\Gamma(\Delta-1) \Gamma\left(\frac{d}{2}-\Delta\right)} G_{d-\Delta, J}^{(a, b)}(z, \bar{z})\right) .
$$

Eqs. (3.24) and (3.26) constitute the main results of this paper: an exact expression for correlators in Regge kinematics, and a corresponding all-order asymptotic expansion in the Regge limit. The latter will be confronted in the next section with explicit expressions in the fishnet model.

\subsection{Formula for double-discontinuity: recovering Lorentzian inversion}

As a first test of eq. (3.24) we will now verify that it is consistent with the Lorentzian inversion formula. The Lorentzian inversion formula extracts the OPE data from the double discontinuity:

$$
c^{t}(\Delta, J)=\frac{\kappa_{\Delta+J}^{(a, b)}}{4} \int_{0}^{1} d z d \bar{z} \mu(z, \bar{z}) G_{J+d-1, \Delta+1-d}^{(-a,-b)}(z, \bar{z}) \operatorname{dDisc}[\mathcal{G}(z, \bar{z})]
$$


where the measure is $\mu=\frac{1}{z^{2} \bar{z}^{2}}\left|\frac{z-\bar{z}}{z \bar{z}}\right|^{d-2}$ and the double-discontinuity is defined as

$$
\begin{aligned}
\operatorname{dDisc}[\mathcal{G}(z, \bar{z})] & \equiv \cos (\pi(a+b)) \mathcal{G}(z, \bar{z})-\frac{1}{2} e^{i \pi(a+b)} \mathcal{G}\left(z, \bar{z}^{\circlearrowleft}\right)-\frac{1}{2} e^{-i \pi(a+b)} \mathcal{G}\left(z, \bar{z}^{\circlearrowright}\right) \\
& =\frac{-i}{2}\left(\operatorname{Disc}_{14}[\mathcal{G}(z, \bar{z})]-\overline{\operatorname{Disc}}_{14}[\mathcal{G}(z, \bar{z})]\right)
\end{aligned}
$$

where the single discontinuity as defined in eq. (2.4) and $\overline{\text { Disc }}$ is the opposite analytic continuation with $i \mapsto-i$. On the other hand, we just obtained an exact formula (3.24) for the discontinuity of the correlator. One might think that the double discontinuity should vanish since $\mathrm{dDisc} \propto \mathrm{Disc}_{23} \operatorname{Disc}_{14}$ which vanishes for any block, however, as stressed below eq. (3.24), the phase $e^{i \pi J}$ is only valid for the counter-clockwise path. It is easy to see from the second form of dDisc that the dDisc is just the imaginary part of that phase, so that the $c^{u}$ term and sine denominator simply cancel out:

$$
\mathrm{dDisc}[\mathcal{G}(z, \bar{z})]=\int_{-\frac{d-2}{2}-i \infty}^{-\frac{d-2}{2}+i \infty} \frac{d J}{2 \pi i} \int_{-\infty}^{\infty} \frac{d \nu}{2 \pi} \frac{c^{t}(\Delta, J)}{2 \kappa_{\Delta+J}^{(a, b)}} R_{\Delta, J}^{(a, b)}(z, \bar{z}) .
$$

This is the main result of this subsection. A similar formula was used recently in a paper involving one of the authors [8], but using only the $G_{1-J, 1-\Delta}^{(a, b)}(z, \bar{z})$ part of the Regge block (3.17), which was valid since that reference only considered the leading power. In contrast, eq. (3.30) is an exact representation.

Following the method above eq. (3.26), eq. (3.30) can be used to obtain asymptotic expansions in the Regge limit. The difference between the formula with the $R$ block and $G$ block is a function for which the $J$ contour can be deformed to the right, and whose purpose is to cancel type-2 spurious poles on the left. Thus eq. (3.30) with $R_{\Delta, J}^{(a, b)}(z, \bar{z}) \mapsto$ $G_{1-J, 1-\Delta}^{(a, b)}(z, \bar{z})$ would only be valid if supplemented by an instruction to discard type-2 spurious poles, and would then define only an asymptotic expansion. The exact integral representation requires the Regge block $R$.

As a check, it is tempting to view eq. (3.30) as the "forward" version of the Lorentzian inversion formula, with the $G$ and $R$ blocks dual to each other. This requires the following pairing to act as an orthogonality relation of sorts:

$$
P_{\Delta, J ; \Delta^{\prime}, J^{\prime}}^{(a, b)} \equiv \frac{\kappa_{\Delta+J}^{(a, b)}}{4} \int_{0}^{1} d z d \bar{z} \mu(z, \bar{z}) G_{J+d-1, \Delta+1-d}^{(-a,-b)}(z, \bar{z}) \frac{R_{\Delta, J}^{(a, b)}(z, \bar{z})}{2 \kappa_{\Delta^{\prime}+J^{\prime}}^{(a, b)}} .
$$

In appendix $\mathrm{D}$ we compute the integral exactly in $d=2$ and $d=4$, using the fact that it factorizes into one-dimensional pairings which we could compute exactly using the Casimir equation satisfied by the blocks. We find that in both dimensions the pairing is given by the following single formula:

$$
\begin{aligned}
P_{\Delta, J ; \Delta^{\prime}, J^{\prime}}^{(a, b)}= & \frac{r_{J+d-\Delta}^{(a, b)}}{r_{J^{\prime}+d-\Delta^{\prime}}^{(a, b)}} \frac{r_{J^{\prime}+\Delta^{\prime}}^{(-a,-b)}}{r_{J+\Delta}^{(-a,-b)}} \frac{4(J+\Delta-1)\left(J^{\prime}+d-\Delta^{\prime}-1\right)}{\left(\Delta-\Delta^{\prime}+J-J^{\prime}\right)\left(\Delta-\Delta^{\prime}-J+J^{\prime}\right)\left(\Delta-\tilde{\Delta}^{\prime}-J+\tilde{J}^{\prime}\right)\left(\Delta-\tilde{\Delta}^{\prime}+J-\tilde{J}^{\prime}\right)} \\
& +\left(\Delta^{\prime} \text { shadow }\right)
\end{aligned}
$$


where $\tilde{\Delta}=d-\Delta$ and $\tilde{J}=2-d-J$ denote the dimension and spin shadows, respectively. The last line is equal to the first with $\Delta^{\prime} \mapsto \tilde{\Delta}^{\prime}$ and multiplied by $K_{\tilde{\Delta}^{\prime}, J^{\prime}}^{(a, b)} / K_{\Delta^{\prime}, J^{\prime}}^{(a, b}$, which is the appropriate relation between the block and its shadow.

It would be interesting to compute eq. (3.32) in other spacetime dimensions. Eq. (3.32) can't be quite the full answer when $d \neq 2,4$, since in these cases it does not transform correctly under either $\Delta$ or $J^{\prime}$ shadow transformations.

Plugging the Regge limit in eq. (3.30) into the Lorentzian inversion formula in eq. (3.28), the pairing should in principle recover the OPE data:

$$
c^{t}(\Delta, J) \stackrel{?}{=} \int_{-\frac{d-2}{2}-i \infty}^{-\frac{d-2}{2}+i \infty} \frac{d J^{\prime}}{2 \pi i} \int_{\frac{d}{2}-i \infty}^{\frac{d}{2}+i \infty} \frac{d \Delta^{\prime}}{2 \pi i} c^{t}\left(\Delta^{\prime}, J^{\prime}\right) P_{\Delta, J ; \Delta^{\prime}, J^{\prime}}^{(a, b)}
$$

Thanks to shadow symmetry of the coefficients $c^{t}\left(\Delta^{\prime}, J^{\prime}\right)$, we can ignore the second line of eq. (3.32) at the cost of a factor 2. In the Lorentzian inversion formula we are supposed to take $J>j_{*}$ so that the integral converges. When $\operatorname{Re}(\Delta)=-d / 2$, there are then no $J^{\prime}$-poles in the right half-plane from $c^{t}\left(\Delta^{\prime}, J^{\prime}\right)$ nor from $r$ functions, and the only singularities are two explicit poles in $P_{\Delta, J ; \Delta^{\prime}, J^{\prime}}^{(a, b)}$. Deforming the $J^{\prime}$ contour to the right we thus get:

$$
\begin{aligned}
c^{t}(\Delta, J) \stackrel{?}{=} \int_{\frac{d}{2}-i \infty}^{\frac{d}{2}+i \infty} \frac{d \Delta^{\prime}}{2 \pi i\left(\Delta-\Delta^{\prime}\right)} & {\left[\frac{r_{J-\Delta+2 \Delta^{\prime}}^{(-a,-b)}}{r_{J+\Delta}^{(-a,-b)}} \frac{J+\Delta-1}{J+\Delta^{\prime}-1} c^{t}\left(\Delta^{\prime}, J-\Delta+\Delta^{\prime}\right)\right.} \\
- & \left.\frac{r_{J+d-\Delta}^{(a, b)}}{r_{J+d+\Delta-2 \Delta^{\prime}}^{(a, b)}} \frac{J+d+\Delta-2 \Delta^{\prime}-1}{J+d-\Delta^{\prime}-1} c^{t}\left(\Delta^{\prime}, J+\Delta-\Delta^{\prime}\right)\right] .
\end{aligned}
$$

We are reduced to a single integral. Note that the two terms in the parenthesis cancel out when $\Delta^{\prime}=\Delta$ so there are no singularities along the integration contour. To perform the integral, we notice that the top line is devoid of singularities in the right half-plane $\operatorname{Re} \Delta^{\prime}>d / 2$, since the coefficient $c^{t}\left(\Delta^{\prime}, J^{\prime}\right)$ is analytic between there and the unitarity bound, and the twist $\Delta^{\prime}-J^{\prime}=\Delta-J$ is held constant (and below the unitarity bound for sufficiently large $J$ ) during integration. Similarly, using the shadow relation between $c^{t}\left(\Delta^{\prime}, J^{\prime}\right)$ and $c^{t}\left(d-\Delta^{\prime}, J^{\prime}\right)$ the second is devoid of poles in the left half-plane in $d=2,4$. Starting from a contour slightly to the left, and deforming the contour in the two lines to the right and left, respectively, we thus pick a single pole from the top line:

$$
\begin{aligned}
c^{t}(\Delta, J) & \stackrel{?}{=}-\operatorname{Res}_{\Delta^{\prime}=\Delta} \frac{1}{\Delta-\Delta^{\prime}} \frac{r_{J-\Delta+2 \Delta^{\prime}}^{(-a,-b)}}{r_{J+\Delta}^{(-a,-b)}} \frac{J+\Delta-1}{J+\Delta^{\prime}-1} c^{t}\left(\Delta^{\prime}, J-\Delta+\Delta^{\prime}\right) \\
& =c^{t}(\Delta, J) .
\end{aligned}
$$

This confirms that eq. (3.30) is precisely dual to the Lorentzian inversion formula, at least in $d=2,4$. It is what the "inversion" (3.28) inverts!

\section{The Lorentzian fishnet model at subleading powers}

The solvability of the fishnet theory provides a testing ground for the results of the preceeding section. On the one hand, the Euclidean solution (2.10) can be analytically continued 


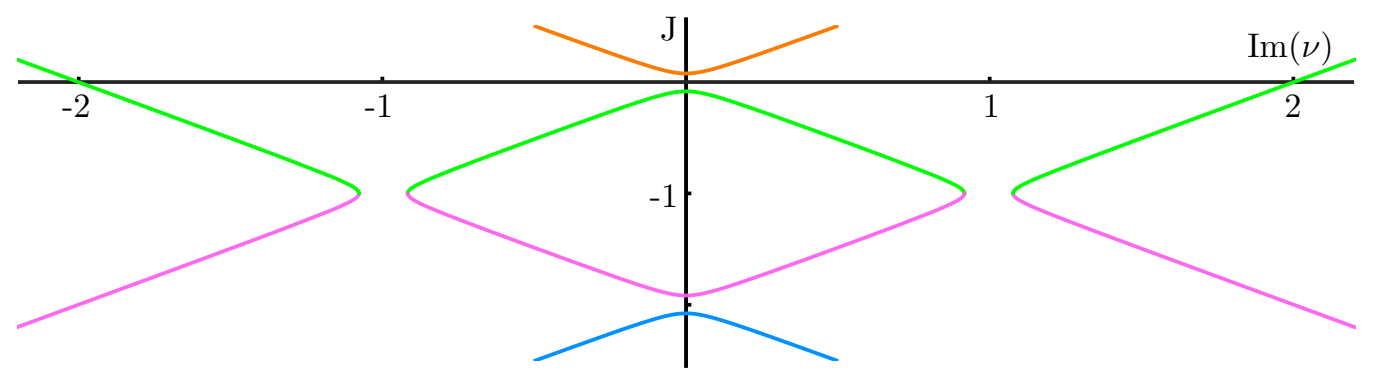

Figure 9. The Regge trajectories in conformal fishnet theory. At zero coupling the trajectories collide on integer spin and scaling dimension. The four colours correspond to the solutions in (4.1), from top along vertical axis: $J_{1}$ is orange, $J_{2}$ is green, $J_{3}$ is pink and $J_{4}$ is blue. Note that $\operatorname{Im}(\nu)=-(\Delta-2)$.

to the Lorentzian regime directly. A nice technique for determining this continuation based on properties of HPL functions is outlined in appendix B. On the other hand, the correlator (2.6) is already written in the spectral decomposition required for the analytic continuation of the conformal blocks. The only challenge after applying the continuation is the navigation of complex $j$ and $\nu$ planes as the integration contours are deformed.

\subsection{The zero-magnon correlator: $u$-channel ladders}

The relevant equations for the zero-magnon correlator were outlined in section 2.2. We reintroduce the shadow block to (2.6) by exploiting the shadow symmetry and compare with equation (3.8). This allows us to extract all the OPE data which can be inserted directly into equation (3.26) for the discontinuity. Since the $u$ - and $t$-channel ladders were computed separately, we treat them separately in this section as well. The $u$-channel ladder contributes only to the $c^{u}$ part of eq. (3.26) due to the $(-1)^{J}$ factor of eq. (2.6).

To compute the discontinuity of the $u$-channel ladders (eq. (2.6)), we first focus on the modified block $G_{1-\Delta, 1-j}^{(0,0)}$ which enters eq. (3.26). As prescribed, we isolate the physical poles in the $j$-plane from the four solutions to eq. (2.9),

$$
J(\nu, \xi)=-1 \pm \sqrt{1-\nu^{2} \pm \sqrt{-\nu^{2}+4 \xi^{4}}}
$$

which are labelled $J_{i}$ for $i=1, \ldots, 4$, illustrated in figure 9 . These solutions correspond to the $j_{n}(\Delta)$ 's discussed in section 3.3.

After evaluating the $j$-residues in eq. (3.26) we only have the $\nu$ integral remaining:

$$
\begin{aligned}
\operatorname{Disc}_{14} \mathcal{G}^{u}(z, \bar{z})= & \sum_{i=1}^{4} \int_{-\infty}^{\infty} d \nu \frac{1}{2 \sin \left(\pi J_{i}\right)} \frac{1}{J_{i}\left(2+J_{i}\right)+\nu^{2}} \Pi_{\Delta, J_{i}} G_{1-\Delta, 1-J_{i}}^{(0,0)}(z, \bar{z}) \\
& +\left(F_{\Delta, J}^{\prime(0,0)} \text { term }\right),
\end{aligned}
$$

where we have absorbed factors from the analytic continuation and normalization into

$$
\Pi_{\Delta, J}=C_{\Delta, J} \frac{2^{2(\Delta+J)} \Gamma\left(\frac{1}{2}(J+\Delta-1)\right) \Gamma\left(\frac{1}{2}(J+\Delta+1)\right)}{2(J+1) \Gamma\left(\frac{J+\Delta}{2}\right)^{2}} .
$$



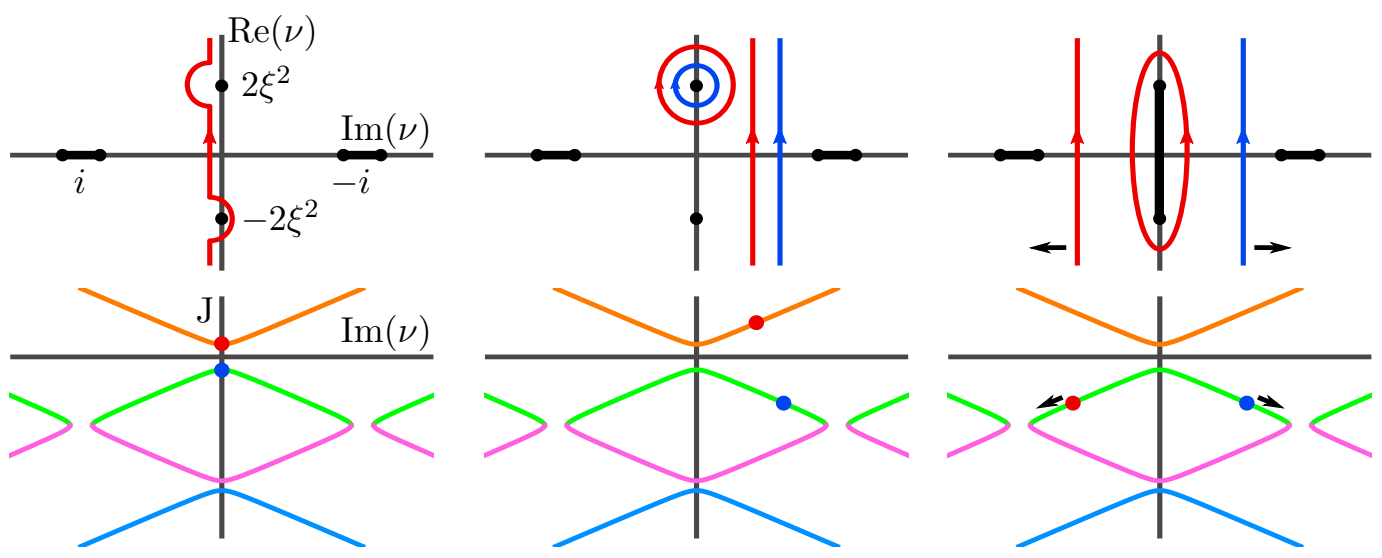

Figure 10. The movement of contours along the $J$ trajectories. The upper panels indicate the $J_{L}$ and $J_{R}$ contours in the $\nu$-plane while the lower panels indicate their corresponding position on the $J$ solution sheets. The strategy to expand in the Regge limit is to move down as fast as possible (so as to $J$ ), as shown in the third column.

where as before $\Delta=2+i \nu$. At lowest order in $\sigma$, only the trajectories with the positive square root, $J_{1}$ and $J_{2}$, will contribute since $G_{1-\Delta, 1-j} \sim \sigma^{1-j}$. The integral over $\nu$ can be evaluated by residues, though the pole and branch structure is significantly more complicated than in the Euclidean case. At subleading powers, all trajectories contribute.

We expand the integrands of (4.2) to a desired order in $\sigma=z \bar{z}$ so the $\nu$ integration becomes manageable. The initial contours for all trajectories run along the real $\nu$-axis, and for $J_{1}$ and $J_{2}$ they go below and above poles at $\nu=-2 \xi^{2}$ and $\nu=2 \xi^{2}$, respectively, since $\operatorname{Im}\left(\xi^{2}\right)<0$. The contours are then deformed as illustrated in figure 10. Each of integrands has a branch cut running between the two poles where the $J_{1}$ and $J_{2}$ sheets intersect. These cuts cancel perfectly when the two integrands are added.

The first step in the integration is to drag both contours to the right, picking up a residue at $\nu=2 \xi^{2}$. In the Regge limit we strive to decrease $J$ (to make the integrand as small as possible) and so we must drag the $J_{1}$ contour back across its branch cut and onto the $J_{2}$ sheet, as drawn in panel 3 of figure 10. Since the labellings no longer refer to the original solutions, we relabel the integrands as $J_{L}$ on the left and $J_{R}$ on the right. The residues at this step contribute to $\operatorname{Disc}_{14} \mathcal{G}^{u}(z, \bar{z})$ at order $\sigma$ and higher. We also find that within an $\mathcal{O}\left(\xi^{4}\right)$ radius each of the branch points and poles shown in figure 10 there are poles from the cosecant function in the $\nu$-plane that must be included in the calculation. They are not included in the plots because in general we expand in small $\xi$ for calculations, at which point the cosecant poles coinside with the plotted solutions. Later on in the calculations the poles from the cosecant will be independent and must then be treated independently.

At leading power, the correlator is thus saturated by the contribution from the branch cut shown in the third column of figure 10 , ranging over $\nu \in\left[-2 \xi^{2}, 2 \xi^{2}\right]$. This phenomenon was observed in refs. $[15,16]$. At subleading powers, we will obtain similar contributions from other intersections, as we now see. 

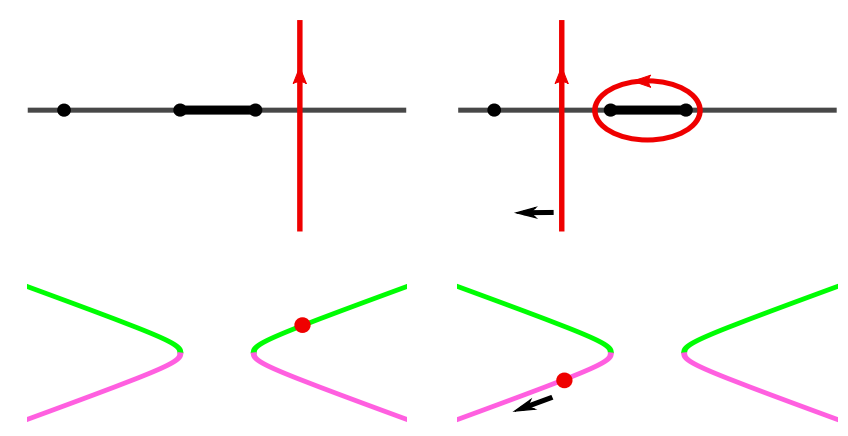

Figure 11. The movement of contours along the $J_{L}$ trajectory past the branch cut around $\nu=i$. As the contour is dragged further to the left along the $J_{3}$ solution it will pick up residues of the cosecant function when $J_{3}(\nu)$ is an integer.
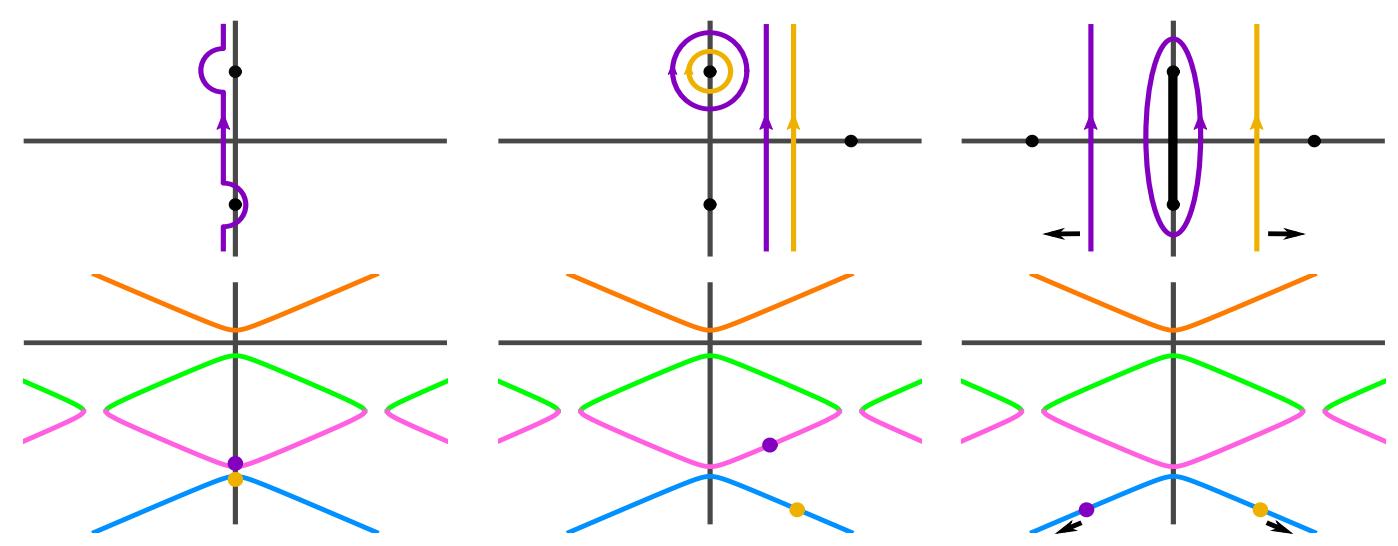

Figure 12. The third intersection integration is analogous to the first. The integration contours will pick up residues at the poles of the cosecant as they are pulled to larger negative $J$.

The next feature that the $J_{L}$ contour encounters is a branch cut running from $\nu=$ $-i-2 \xi^{2}-\mathcal{O}\left(\xi^{4}\right)$ to $\nu=-i+2 \xi^{2}+\mathcal{O}\left(\xi^{4}\right)$. This branch is analogous to the first but for the $J_{2}$ and $J_{3}$ intersection. The contribution to the integrand at this location is the contour around the branch cut, as illustrated in figure 11. The $J_{L}$ contour can now be dragged to imaginary infinity in the $\nu$-plane with the only obstructions being poles of the cosecant at integer $j$. The first of these is at $J_{3}=-2$ and hence contributes at $\sigma^{3}$. Due to the $\nu \leftrightarrow-\nu$ symmetry of the integrand, the computation for the right-moving contour is equivalent up to signs from contour orientations. Since $J=-1$ around these branches, these residues contribute to $\operatorname{Disc}_{14} \mathcal{G}^{u}(z, \bar{z})$ at order $\sigma^{2}$.

Also at order $\sigma^{3}$, we must consider the $J_{3}$ and $J_{4}$ trajectories at $J=-2$ in the same way as the $J=0$ intersection. This contour deformation is illustrated in figure 12. Additional contributions to the correlation function at $\sigma^{4}$ come from the poles of the cosecant function along the $J_{3}$ and $J_{4}$ trajectories, arising from all the contours being pulled to more negative $J$. These additional points are plotted in figure 13 .

To summarize, the $\nu$ integration contour was deformed along the Regge trajectories while collecting contours around poles and branches at the $J=0$ intersection which start 


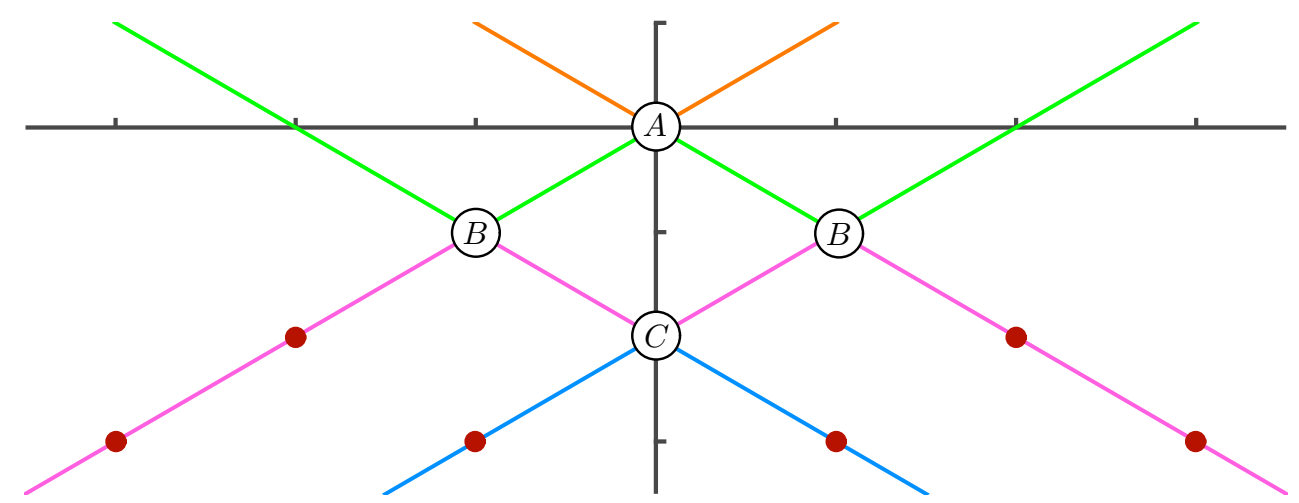

Figure 13. Contributions to the Regge-limit four-point correlator of Conformal Fishnet Theory. Cuts $A-C$ occur at the intersections of the trajectories and the calculations correspond to figures 10, 11 and 12. The additional maroon dots occur at the poles of the cosecant function. As hoped, all terms come from localized points or short cuts on the Regge trajectories.

contributing to $\operatorname{Disc}_{14} \mathcal{G}^{u}(z, \bar{z})$ at order $\sigma$, branch contours at $J=-1$ of order $\sigma^{2}$ and pole and branch contours at $J=-2$ of order $\sigma^{3}$.

The $F_{J, \Delta}^{\prime(0,0)}(z, \bar{z})$ term is the remaining contributor to the discontinuity (4.2). It can be evaluated in the same manner as the Euclidean four-point function but with an additional coefficient from the $s_{\beta}^{(0,0)}$ term in eq. (3.26), which in this case reduces to

$$
G_{\Delta, J}^{(0,0)} \rightarrow-\tan \left(\frac{\pi}{2}(J+\Delta)\right) G_{\Delta, J}^{(0,0)} .
$$

We can now present the various contour integration results up to $\sigma^{3}$ and to the first two orders of $\xi^{2}$. The corresponding locations on the $\nu$-contours are indicated in figure 13 . We computed these terms along with additional residues at the poles of the cosecant function up to orders $\left(\sigma^{4}, \xi^{8}\right)$ and $\left(\sigma^{3}, \xi^{12}\right)$. We found perfect agreement with the direct HPL continuations; to illustrate the nontrivial interplay between the contributions, we now record explicit formulas at lower order.

The first intersection $(\nu=0, J=0)$ contributes:

$$
\begin{aligned}
i A= & \sigma\left(-\frac{4 \pi \xi^{2} w \log (w)}{(w-1)(w+1)}-\frac{4 i \pi \xi^{4} w(\log (\sigma)-1) \log (w)}{(w-1)(w+1)}\right) \\
& +\sigma^{2}\left(-\pi \xi^{2}-\frac{i \pi \xi^{4}\left(-\log (\sigma)+w^{2} \log (\sigma)-w^{2}+w^{2} \log (w)+\log (w)+1\right)}{(w-1)(w+1)}\right) \\
& +\sigma^{3}\left(-\frac{\pi \xi^{2}\left(w^{2}+1\right)}{2 w}-\frac{i \pi \xi^{4}\left(-\log (\sigma)+w^{4} \log (\sigma)-w^{4}+w^{4} \log (w)+\log (w)+1\right)}{2(w-1) w(w+1)}\right) \\
& +\mathcal{O}\left(\sigma^{4}, \xi^{6}\right) .
\end{aligned}
$$

The second intersection $(\nu= \pm i, J=-1)$ :

$$
\begin{aligned}
i B= & \sigma^{2}\left(\frac{i \pi \xi^{4}\left(\log (\sigma)-w^{2} \log (\sigma)+w^{2}+w^{2} \log (w)+\log (w)-1\right)}{(w-1)(w+1)}\right) \\
& +\sigma^{3}\left(\frac{i \pi \xi^{4}\left(\log (\sigma)-w^{4} \log (\sigma)+w^{4}+w^{4} \log (w)+\log (w)-1\right)}{2(w-1) w(w+1)}\right)+\mathcal{O}\left(\sigma^{4}, \xi^{6}\right) .
\end{aligned}
$$


The third Intersection $(\nu=0, J=-2)$ :

$$
i C=\sigma^{3}\left(\frac{\pi \xi^{6} w \log (w)}{(w-1)(w+1)}+\frac{i \pi \xi^{8} w(\log (\sigma)-2) \log (w)}{(w-1)(w+1)}\right)+\mathcal{O}\left(\sigma^{4}, \xi^{10}\right) .
$$

The cosecant poles $(\nu= \pm 2 i, J=-2)$ :

$$
i D=\sigma^{3}\left(-\frac{i \pi \xi^{4}\left(w^{2}+1\right)}{4 w}-\frac{i \pi \xi^{8}\left(-11 w^{4}+4 w^{4} \log (w)+4 \log (w)+11\right)}{16(w-1) w(w+1)}\right)+\mathcal{O}\left(\sigma^{4}, \xi^{10}\right) .
$$

Finally, the $F_{J, \Delta}^{(0,0)}(z, \bar{z})$ contribution:

$$
\begin{aligned}
i E= & \sigma^{2}\left(\pi \xi^{2}+2 i \pi \xi^{4}(\log (\sigma)-1)\right) \\
& +\sigma^{3}\left(\frac{\pi \xi^{2}\left(w^{2}+1\right)}{2 w}+\frac{i \pi \xi^{4}\left(w^{2}+1\right)(4 \log (\sigma)-3)}{4 w}\right)+\mathcal{O}\left(\sigma^{4}, \xi^{6}\right) .
\end{aligned}
$$

The sum of these expressions $A-E$ is then found to be equal to the $\operatorname{Disc}_{14}$ of the fishnet correlation function given in equation (2.10), after the analytic continuation of harmonic polylogarithms detailed in appendix B:

$$
\begin{aligned}
\operatorname{Disc}_{14} \mathcal{G}^{u}(z, \bar{z})= & \sigma\left(\frac{4 i \pi \xi^{2} w \log (w)}{(w-1)(w+1)}-\frac{4 \pi \xi^{4} w(\log (\sigma)-1) \log (w)}{(w-1)(w+1)}\right. \\
& \left.-\frac{2 i \pi \xi^{6} w \log (w)\left(4 \log ^{2}(w)+2 \pi^{2}-9\right)}{3(w-1)(w+1)}\right) \\
& +\frac{1}{3} \sigma^{2} \xi^{6}\left(-4 \log ^{3}(\sigma)+6 i \pi \log ^{2}(\sigma)+12 \log ^{2}(\sigma)-12 i \pi \log (\sigma)\right. \\
& \left.-15 \log (\sigma)-6 i \pi \log ^{2}(w)-12 \zeta(3)+12 i \pi+15\right)+\mathcal{O}\left(\sigma^{3}, \xi^{8}\right) .
\end{aligned}
$$

\section{2 $t$-channel ladders and their double discontinuity}

The Regge limit can also be considered for the $t$-channel ladders, for which the Euclidean OPE is given by the same expression as eq. (2.6) but without the overall $(-1)^{J}$ factor. The $t$-channel data is interesting because it is the only contributor to $\operatorname{dDisc} \mathcal{G}(z, \bar{z})$ (see eq. (3.30)). We checked that the Sommerfeld-Watson calculation matched the direct HPL continuation up to order $\sigma^{2}$ and $\xi^{8}$, this time including the $e^{i \pi J}$ factor in eq. (3.26). At this level, we would have detected any errors in the formula for the analytic continuation that would not have been sensed in the $u$-channel case. The $t$-channel HPL continutations were obtained from the $u$-channel results by substituting $z \rightarrow z /(z-1)$ and $\bar{z} \rightarrow \bar{z} /(\bar{z}-1)$ with appropriate phases.

In section 3 we presented equation (3.30) for the double discontinuity in terms of a double integral of the OPE data and the Regge block over spin and scaling dimension, which we also checked explicitly using the $t$-channel ladders the fishnet model. The calculations are performed almost identically to those for the Disc ${ }_{14}$, however the cosecant function in spin is removed along with several constant coefficients from definitions. For the $G_{1-\Delta, 1-J}$ block, the analytic structure and $\nu$ integration follows the contour deformations drawn in figures 10, 11 and 12. The contributions from the poles at $\nu= \pm 2 \xi^{2}$ vanish and therefore 
the double discontinuity contains only terms at even powers of $\xi^{2}$. Moreover, there are no longer poles from a cosecant contributing at orders $\sigma^{3}$ and higher. In reference to figure 13, only terms from locations $A-C$ contribute. As before, the remaining shadow blocks are irrelevant for the calculation.

We verified that equation (3.30) was correct for the zero-magnon four-point function by comparing the direct integration of the (3.30) to the analytic continuations of the HPL functions appearing in the first line of (3.29). We computed the double discontinuity to orders $\left(\sigma^{4}, \xi^{12}\right)$ in both ways and found perfect agreement. In the Regge limit, the double discontinuity is

$$
\begin{aligned}
\operatorname{dDisc}[\mathcal{G}(z, \bar{z})]= & \sigma\left(\frac{4 \pi^{2} \xi^{4} w \log (w)}{(w-1)(w+1)}-\frac{2 \pi^{2} \xi^{8} w \log (w)\left(-3 \log ^{2}(\sigma)+3 \log ^{2}(w)+\pi^{2}\right)}{3(w-1)(w+1)}\right) \\
& +\sigma^{2} \frac{2 \pi^{2} \xi^{8}}{(w-1)(w+1)}\left(-\log ^{2}(\sigma)+\log (\sigma)+w^{2} \log ^{2}(\sigma)-w^{2} \log (\sigma)\right. \\
& \left.-w^{2} \log ^{2}(w)+w^{2} \log (w)+\log ^{2}(w)+\log (w)\right)+\mathcal{O}\left(\sigma^{3}, \xi^{12}\right) .
\end{aligned}
$$

\subsection{The one-magnon correlator}

We can follow the main steps of the zero-magnon case to compute the Regge limit of the one-magnon four-point function. The interest is that the external operators have varying scaling dimensions, namely $\Delta_{1}=\Delta_{4}=2$ and $\Delta_{2}=\Delta_{3}=1$, so it will allow us to further verify the equations of section 3 ( $a$ and $b$ vanished in the zero-magnon case and are now non-zero, $a=b=-1 / 2$ ). Interestingly, the Regge trajectories are significantly simpler! Moreover, when computing contributions to the Regge limit, there is only one branch cut to worry about (which contributes at leading order) and the only additional features leading to subleading corrections include the poles of the cosecant function attached to the $G_{1-J, 1-\Delta}$ block and sum over spins in the $F_{\Delta, J}^{\prime}$ block. Since the formulae for the analytic continuations are relevant at the leading order ( $\sigma^{2}$ in this case) and first subleading order (now $\sigma^{3}$ ), we compute Disc 14 of the one-magnon correlator only to order $\sigma^{3}$ and $\xi^{6}$. We again find agreement with known fishnet data once analytically continued to the Lorentzian regime and evaluated at high energy.

We first review the physics of the Euclidean one-magnon four-point function derived in [14] and sketched in figure 14. The correlator is given by (again, trace implied)

$$
\left\langle 0\left|Y\left(x_{1}\right) X\left(x_{1}\right) Y\left(x_{2}\right) Y^{\dagger}\left(x_{3}\right) Y^{\dagger}\left(x_{4}\right) X^{\dagger}\left(x_{4}\right)\right| 0\right\rangle \equiv \frac{\left(x_{13}^{2} x_{24}^{2}\right)^{1 / 2}}{x_{14}^{2}\left(x_{12}^{2} x_{34}^{2}\right)^{3 / 2}} \mathcal{G}_{1}(z, \bar{z})
$$

The sum over conformal blocks is slightly modified to

$$
\mathcal{G}_{1}(z, \bar{z})=\sum_{J \geq 0} \int_{-\infty}^{\infty} \frac{d \nu}{2 \pi} C_{\Delta, J}^{\prime} \frac{256(-1)^{J} H_{\Delta, J}^{2}}{1-16 \xi^{2} H_{\Delta, J}} G_{\Delta, J}^{\left(-\frac{1}{2},-\frac{1}{2}\right)}(z, \bar{z}),
$$

with a new set of energy eigenvalues,

$$
H_{\Delta, J}=\frac{(-1)^{J}}{4(-\Delta+J+3)(\Delta+J-1)} .
$$




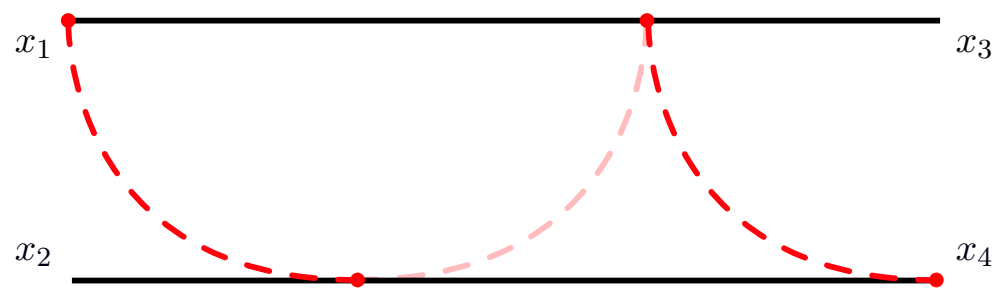

Figure 14. An example of a fishnet ladder diagram evaluated in the computation of $\mathcal{G}(z, \bar{z})$ with one-magnon operators. The four-point interaction sites have coupling $\xi^{2}$ so this diagram contributes at order $\xi^{4}$ and the shading of the propagator indicates how it "winds". The points $x_{1}$ and $x_{2}$ "source" the propagator and account for the modified scaling dimensions of the operators inserted at these points (see [14] for additional diagrams and discussion).

A novelty is that $(-1)^{J}$ occurs both in the numerator and denominator of eq. (4.13); as remarked in [14], its appearance in $H$ is necessary to cancel the spurious poles of the blocks with the spurious poles of the normalization coefficient, which in this case includes the external operator dimensions [14]

$$
\begin{aligned}
C_{\Delta, J}^{\prime}= & \frac{\Gamma(\Delta-1) \Gamma(2+J) \Gamma(4-\Delta+J)}{2 \Gamma(J+1) \Gamma(\Delta-2) \Gamma(\Delta+J-1)} \\
& \times \frac{\Gamma\left(\frac{1}{2}\left(\Delta+J+\Delta_{1}-\Delta_{2}\right)\right) \Gamma\left(\frac{1}{2}\left(\Delta+J-\Delta_{1}+\Delta_{2}\right)\right)}{\Gamma\left(2-\frac{1}{2}\left(\Delta-J-\Delta_{1}+\Delta_{2}\right)\right) \Gamma\left(2-\frac{1}{2}\left(\Delta-J+\Delta_{1}-\Delta_{2}\right)\right)} .
\end{aligned}
$$

Just as in the zero-magnon case, the correlator can be expanded in the coupling,

$$
\mathcal{G}_{1}(z, \bar{z})=\frac{(z \bar{z})^{3 / 2}}{z-\bar{z}} \sum_{n=0}^{\infty} \xi^{2 n} \mathcal{G}_{1}^{(n)}(z, \bar{z})
$$

and the $\mathcal{G}_{1}^{(n)}$ 's are combinations of HPLs. At leading order we have

$$
\mathcal{G}_{1}^{(0)}(z, \bar{z})=z-\bar{z} .
$$

We verified the expansions from [14] to order $\sigma^{4}$ and $\xi^{8}$.

We now wish to analytically continue our correlator (4.13) to the Lorentzian kinematics regime. To make contact with eq. (3.21), one can work out that

$$
\begin{aligned}
c^{t}(\Delta, J) & =\frac{1}{2}\left(c^{\text {even }}(\Delta, J)+c^{\text {odd }}(\Delta, J)\right) \\
c^{u}(\Delta, J) & =\frac{1}{2}\left(c^{\text {even }}(\Delta, J)-c^{\text {odd }}(\Delta, J)\right),
\end{aligned}
$$

where $c^{\text {even }}$ and $c^{\text {odd }}$ represent the OPE data of the correlator (4.13) with even and odd spin, respectively, that is, with $(-1)^{J}$ set to \pm 1 . Unlike the zero-magnon case, both channels contribute to the discontinuity $\operatorname{Disc}_{14} \mathcal{G}_{1}$. The Regge trajectories were computed by solving for the physial poles of the correlation function and are plotted in figure 15 . We denote them

$$
J_{ \pm}^{\text {even }}(\nu, \xi)=-1 \pm \sqrt{-\nu^{2}+4 \xi^{2}} \text { and } J_{ \pm}^{\text {odd }}(\nu, \xi)=-1 \pm \sqrt{-\nu^{2}-4 \xi^{2}}
$$



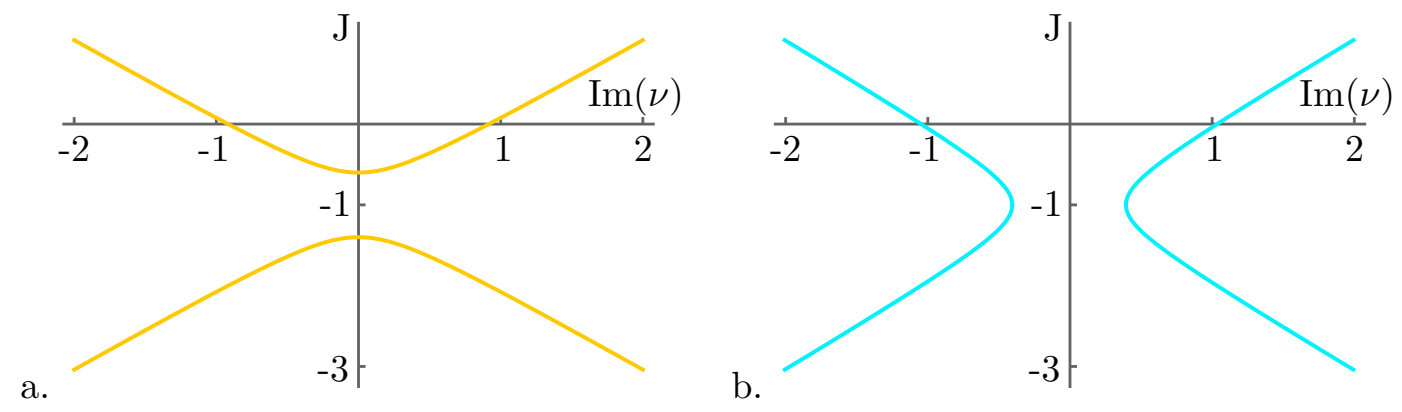

Figure 15. Regge trajectories for the one-magnon four-point function. The $J^{\text {even }}$ and $J^{\text {odd }}$ trajectories are in the left and right panels, respectively. Note that the gap between the curves has length of order $\xi^{2}$.

We can now plug our OPE data into our main equation (3.26), in which our Regge trajectories take the place of the $j_{n}(\nu)$ 's. We have

$$
\begin{aligned}
\operatorname{Disc}_{14} \mathcal{G}_{1}= & \int_{-\infty}^{\infty} d \nu \sum_{j_{n}(\nu)} \operatorname{Res}_{J=j_{n}(\nu)} \frac{1}{\sin (\pi J)} \Pi_{1}(\Delta, J) G_{1-\Delta, 1-J}^{\left(-\frac{1}{2},-\frac{1}{2}\right)}(z, \bar{z}) \\
& +\left(F^{\prime\left(-\frac{1}{2},-\frac{1}{2}\right)}(z, \bar{z}) \text { term }\right)
\end{aligned}
$$

where we collected the OPE data into

$$
\begin{aligned}
\Pi_{1}(\Delta, J)= & C_{\Delta, J}^{\prime} \frac{2^{2 \Delta+2 J} \Gamma\left(\frac{J}{2}+\frac{\Delta}{2}\right)^{2}}{2(-\Delta+J+3) \Gamma\left(\frac{J}{2}+\frac{\Delta}{2}+\frac{1}{2}\right)^{2}} \\
& \times \frac{\left(4 e^{i \pi J} \xi^{2}+(J+1)^{2}+\nu^{2}\right)}{\left((J+1)^{2}+\nu^{2}-4 \xi^{2}\right)\left((J+1)^{2}+\nu^{2}+4 \xi^{2}\right)} .
\end{aligned}
$$

The $e^{i \pi J}$ clearly distinguishes the $t$ - and $u$-channel data. The $\nu$ integration proceeds very much like the zero-magnon case. Since the Regge intercepts is now $J=-1$ (see figure 15), the leading order physics comes at order $\sigma^{1-J_{\max }}=\sigma^{2}$. The analytic structure at $J=-1$ is similar to the zero-magnon cases except that instead of poles at the end of the branch cuts, we find only branch points. Thus the contribution at leading order comes only from the residue around the branch cut. For the $J^{\text {even }}$ trajectories, this branch runs from $-2 \xi^{2}$ to $2 \xi^{2}$ and the integration contours are deformed similarly to figure 10 . For the $J^{\text {odd }}$ trajectories, the branch runs from $-2 i \xi^{2}$ to $2 i \xi^{2}$ and the contours look more like those in figure 11. The movement of these contours is plotted in figure 16 .

At subleading orders in $\sigma$ we have to take into account the poles of the cosecant function. Only the integrands involving the $J^{\text {even }}$ solution contribute at this order.

Additional subleading terms come from the block whose integration contour wasn't deformed. As in the zero-magnon case, we simply have to add a coefficient to the integrand of the Euclidean case to compute the $F^{\prime\left(-\frac{1}{2},-\frac{1}{2}\right)}(z, \bar{z})$ contribution, which becomes

$$
G_{\Delta, J}^{\left(-\frac{1}{2},-\frac{1}{2}\right)} \rightarrow-\cot \left(\frac{\pi}{2}(J+\Delta)\right) G_{\Delta, J}^{\left(-\frac{1}{2},-\frac{1}{2}\right)} .
$$



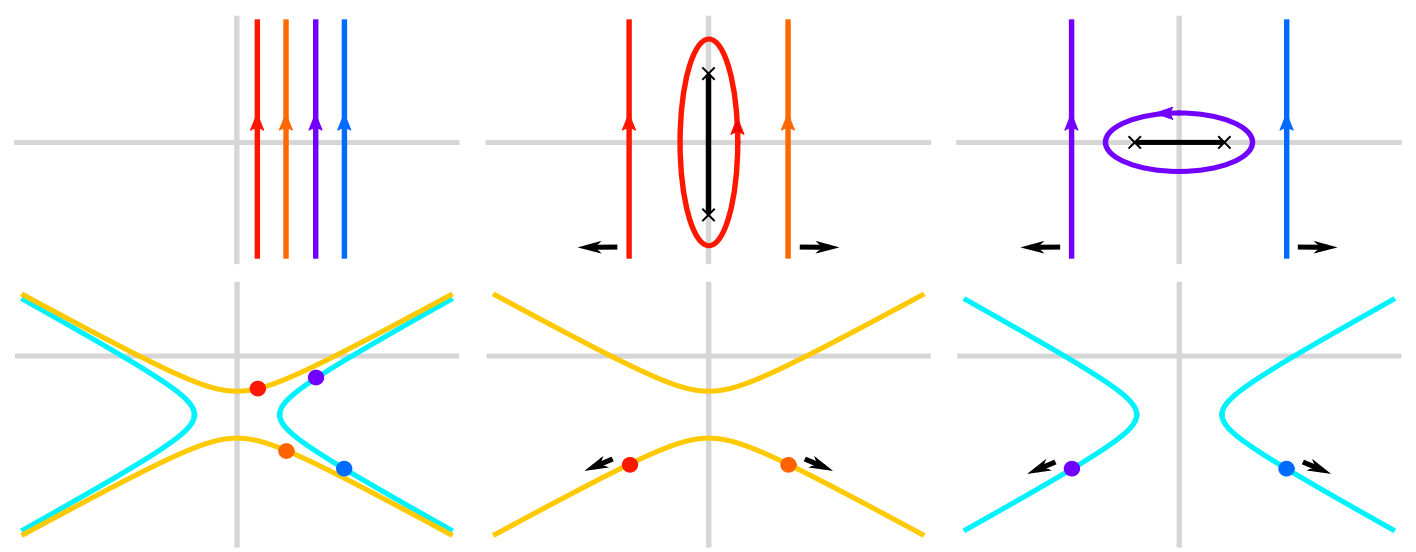

Figure 16. A plot of the $\nu$ integration along the Regge trajectories, following figure 10. The warm and cool colours correspond to the $J^{\text {even }}$ and $J^{\text {odd }}$ solutions, respectively.

We can now present the results of the one-magnon calculations at order $\sigma^{3}$ and the first two orders of $\xi$. We checked to orders $\left(\sigma^{3}, \xi^{6}\right)$ that these contributions matched the direct HPL continuations.

The first intersection along $J^{\text {even }}(\nu=0, J=-1)$ :

$$
\begin{aligned}
i A^{\text {even }}= & \sigma^{2}\left(\frac{\pi^{2} \xi^{4} w(2 \log (\sigma)-i \pi) \log (w)}{(w-1)(w+1)}\right. \\
& \left.+\frac{\pi^{2} \xi^{6} w \log (w)\left(4 \log ^{3}(\sigma)-6 i \pi \log ^{2}(\sigma)-4 \log (\sigma) \log ^{2}(w)+2 i \pi \log ^{2}(w)-24 \zeta(3)-i \pi^{3}\right)}{6(w-1)(w+1)}\right) \\
& +\sigma^{3}\left(\pi^{2} \xi^{4}+\frac{\pi^{2} \xi^{6}}{(w-1)(w+1)}\left(-\log ^{2}(\sigma)+i \pi \log (\sigma)+2 \log (\sigma w)+w^{2} \log ^{2}(\sigma)\right.\right. \\
& \left.\left.-i \pi w^{2} \log (\sigma)-2 w^{2} \log (\sigma / w)+i \pi w^{2}-w^{2} \log ^{2}(w)+\log ^{2}(w)-i \pi\right)\right) \\
& +\mathcal{O}\left(\sigma^{4}, \xi^{8}\right) .
\end{aligned}
$$

The first intersection along $J^{\text {odd }}(\nu=0, J=-1)$ :

$$
\begin{aligned}
i A^{\text {odd }}= & \sigma^{2}\left(\frac{4 i \pi \xi^{2} w \log (w)}{(w-1)(w+1)}-\frac{\pi \xi^{4} w \log (w)\left(2 i \log ^{2}(\sigma)+2 \pi \log (\sigma)-2 i \log ^{2}(w)-i \pi^{2}\right)}{(w-1)(w+1)}\right) \\
& -\sigma^{3} \frac{\pi \xi^{4}\left(-2 i \log (\sigma)+2 i w^{2} \log (\sigma)+\pi w^{2}-2 i w^{2} \log (w)-2 i \log (w)-\pi\right)}{(w-1)(w+1)} \\
& +\mathcal{O}\left(\sigma^{4}, \xi^{6}\right) .
\end{aligned}
$$

The cosecant poles (only from $\left.J^{\text {even }}\right)(\nu= \pm i, J=-2)$ :

$$
i B=\sigma^{3}\left(i \pi \xi^{2}-\frac{i \pi \xi^{4}\left(-3 w^{2}+2 w^{2} \log (w)+2 \log (w)+3\right)}{(w-1)(w+1)}\right)+\mathcal{O}\left(\sigma^{4}, \xi^{6}\right) .
$$

The $F^{\prime\left(-\frac{1}{2},-\frac{1}{2}\right)}(z, \bar{z})$ contribution:

$$
i C=\sigma^{3}\left(-i \pi \xi^{2}+i \pi \xi^{4}(2 \log (\sigma)-3)\right)+\mathcal{O}\left(\sigma^{4}, \xi^{6}\right) .
$$


The sum of these results match the continued HPLs! The full discontinuity in the Regge limit is

$$
\begin{aligned}
\operatorname{Disc}_{14} \mathcal{G}_{1}(z, \bar{z})= & \sigma^{2}\left(\frac{4 \pi \xi^{2} w \log (w)}{(w-1)(w+1)}+\frac{2 \pi \xi^{4} w \log (w)(\log (w)-\log (\sigma))(\log (\sigma)+\log (w))}{(w-1)(w+1)}\right) \\
& +\mathcal{O}\left(\sigma^{4}, \xi^{6}\right) .
\end{aligned}
$$

\section{Conclusion}

This paper extended the formalism of Regge theory applied to four-point correlation functions in conformal field theories. Our main result, eq. (3.24) provides an exact expression for the resummed OPE in a Lorentzian spacetime, which can be expanded at high energies according to eq. (3.26) to compute subleading power corrections in a given model. At leading power, the formula reproduces existing work from the conformal bootstrap literature. The key new ingredient is the Regge block $R$ defined in eq. (3.17), which allows to seamlessly deal with subleading powers. We also obtained an exact representation for the expectation value of a double commutator, eq. (3.30).

The second goal of this paper was to check eq. (3.26) explicitly in conformal fishnet theory, a treasure trove of data. We found perfect agreement to high orders in energy and the coupling in both the zero- and one-magnon four-point functions.

As mentioned in introduction, we expect this formula to be useful in situations which require going beyond the single-exchange approximation, such as situations involving saturation or for precision studies in theories where forward scattering is asymptotically transparent.

\section{Acknowledgments}

Work of SCH is supported by the National Science and Engineering Council of Canada, the Canada Research Chair program, the Fonds de Recherche du Québec-Nature et Technologies, and the Simons Collaboration on the Nonperturbative Bootstrap. JS gratefully acknowledges support from the Institut des Sciences Mathématiques.

\section{A Conformal blocks}

This appendix defines the conformal blocks as used in this paper. For the fishnet theory we require the $d=4$ blocks, which are given explicitly as

$$
G_{\Delta, J}^{(a, b)}(z, \bar{z})=\frac{z \bar{z}}{\bar{z}-z}\left[k_{\Delta-J-2}^{(a, b)}(z) k_{\Delta+J}^{(a, b)}(\bar{z})-(z \leftrightarrow \bar{z})\right] \quad(d=4)
$$

where

$$
k_{\beta}^{(a, b)}(z)=z^{\beta / 2}{ }_{2} F_{1}\left(\frac{\beta}{2}+a, \frac{\beta}{2}+b, \beta, z\right)
$$

is an eigenfunction of the $\mathrm{SL}(2, \mathrm{R})$ Casimir. In general, conformal blocks are eigenfunctions of the conformal Casimir which we normalize so that $z, \bar{z} \rightarrow 0$ limit contains the following term with unit coefficient:

$$
\lim _{z \ll \bar{z} \ll 1} G_{\Delta, J}^{(a, b)}(z, \bar{z})=z^{\frac{\Delta-J}{2}} \bar{z}^{\frac{\Delta+J}{2}} .
$$


This term then comes with an infinite tower of integer powers of $z, \bar{z}$. When the spin is non-integer, this tower is supplemented with a second one such that the combined limit $z, \bar{z} \rightarrow 0$ is a function $C$ proportional to the Gegenbauer function $C_{J}^{(d / 2-1)}(x)$ :

$$
\lim _{z, \bar{z} \rightarrow 0} G_{\Delta, J}^{(a, b)}(z, \bar{z})=(z \bar{z})^{\frac{\Delta}{2}} C_{J}\left(\frac{z+\bar{z}}{2 \sqrt{z \bar{z}}}\right),
$$

where

$$
C_{J}(x)=\frac{\Gamma\left(\frac{d-2}{2}\right) \Gamma(J+d-2)}{\Gamma(d-2) \Gamma\left(J+\frac{d-2}{2}\right)}{ }_{2} F_{1}\left(-J, J+d-2, \frac{d-1}{2}, \frac{1-x}{2}\right) .
$$

The normalization was chosen to be compatible with eq. (A.3).

\section{B Harmonic polylogarithms and their analytic continuations}

Harmonic polylogarithms (HPLs) are generalized logarithms defined and indexed such that

$$
H_{1}(z)=\int_{0}^{z} \frac{d \tilde{z}}{1-\tilde{z}}=-\log (1-z)
$$

and

$$
H_{0}(z)=\int_{0}^{z} \frac{d \tilde{z}}{\tilde{z}}=\log (z) .
$$

Higher-weight HPLs are nested integrals with a binary vector labelling the differential form such that 0 indicates $d \tilde{z} / \tilde{z}$ and 1 indicates $d \tilde{z} /(1-\tilde{z})$, as illustrated in the following example [27]:

$$
H_{0,1,1}(z)=\int_{0}^{z} \frac{d z^{\prime}}{z^{\prime}} \int_{0}^{z^{\prime}} \frac{d z^{\prime \prime}}{1-z^{\prime \prime}} \int_{0}^{z^{\prime \prime}} \frac{d z^{\prime \prime \prime}}{1-z^{\prime \prime \prime}}
$$

It is important to note that HPL functions with a 0 as the rightmost index are singular as $\log (z)^{n}$ at $z=0$ and those with a 1 at the leftmost index are singular as $\log (1-z)^{n}$ at $z=1$. This will be a crucial fact in the subsequent section. These divergences can be extracted by considering the integral representation and expanding according to

$$
H_{a_{1}, \ldots, a_{k}}(z) H_{0}(z)=H_{a_{1}, \ldots, a_{k}, 0}(z)+H_{a_{1}, \ldots, a_{k-1}, 0, a_{k}}(z)+\ldots+H_{0, a_{1}, \ldots, a_{k}}(z) .
$$

Then, by solving for $H_{a_{1}, \ldots, a_{k}, 0}(z)$ and recalling equation (B.2), the divergent logarithm is evident. A similar technique allows for the extraction of the $\log (1-z)$ terms.

It is also interesting to note the relation between HPLs and zeta values, namely that $H_{0, \ldots, 0,1}(1)=\zeta(n)$, where $n$ is the number of 0 indices and $\zeta$ denotes the Riemann zeta function. In a generalization of this relation, the multiple zeta values (MZV) are defined as

$$
\mathrm{MZV}_{a_{1}, \ldots a_{k}}=H_{a_{1}, \ldots a_{k}}(1) \text {. }
$$

For the correlation function of the conformal fishnet theory in equation (2.10), the analytic continuation of $z$ counterclockwise around $z=1$ amounts to extracting the $\log (1-$ $z$ ) terms from the HPL functions and replacing them with the additional contribution from moving around the branch cut, $\log (1-z) \rightarrow 2 \pi i+\log (1-z)$. 


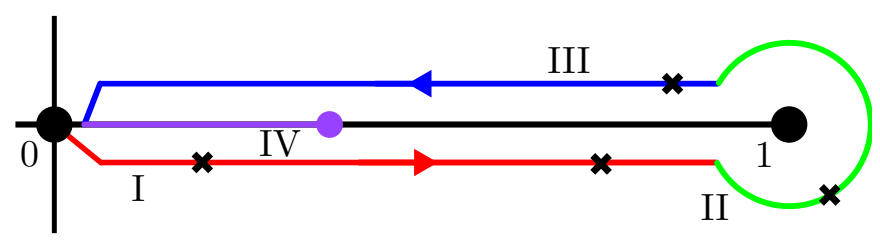

Figure 17. Contour of integration in the complex $z$-plane for the calculation of the $C_{\left\{a_{i}\right\}}$ terms. The $\mathbf{x}$ 's denote the endpoint $z$ position of the integral $\int_{0}^{z}$. Due to the nesting, these $x$ 's are ordered. The integral is evaluated by dividing the contour into parts I, II and III. The remaining section, IV, is the original HPL.

Alternatively, we can compute the analytic continuation of the HPLs without extracting the logarithms by directly evaluating their analytic continuation. From the integral form of the HPL functions (B.3), their analytic continuation can be decomposed as

$$
H_{a_{1}, \ldots, a_{k}}^{\prime}(z)=H_{a_{1}, \ldots, a_{k}}(z)+C_{a_{1}} H_{a_{2}, \ldots, a_{k}}(z)+\ldots+C_{a_{1}, \ldots, a_{k-1}} H_{a_{k}}(z)+C_{a_{1}, \ldots, a_{k}},
$$

where the $C_{\left\{a_{i}\right\}}$ 's denote constant contour integrals starting at the $z$-plane origin and looping counterclockwise around $z=1$. These are illustrated in figure 17 and can be decomposed into integrals from $z=0 \rightarrow z=1$, around a countour at $z=1$ and then from $z=1 \rightarrow z=0$. For example,

$$
C_{1,0,1}=(-1)^{3} \overline{\mathrm{MZV}}_{1,0,1}-2 \pi i \overline{\mathrm{MZV}}_{1,0}-2 \pi i \mathrm{MZV}_{0,1}+\mathrm{MZV}_{1,0,1},
$$

where $\overline{\mathrm{MZV}}$ denotes the MZV value with the binary indices flipped (for example $\overline{\mathrm{MZV}}_{1,0,1}=$ $\left.\mathrm{MZV}_{0,1,0}\right)$. The additional $(-1)^{3}$ term comes from a change of variables in the integral and the $2 \pi i$ comes from the countour integral. In general, these $C_{\left\{a_{i}\right\}}$ constants are calculated by summing all divisions the HPL integrals into the three regions of integration. The middle contour region returns $(-2 \pi i)^{n} / n$ ! if all the indices are 1's and zero otherwise. The example above was calculated by considering the integration regions of $C_{1,0,1}$,

$$
\begin{aligned}
C_{1,0,1}= & C_{101||}+C_{10|1|}+C_{1|01|}+C_{10|| 1}+C_{|101|}+C_{1|0| 1}+C_{1|| 01} \\
& +C_{|10| 1}+C_{|1| 01}+C_{|| 101},
\end{aligned}
$$

where the l's denote these separated regions, the red indicates terms that vanish and the blue indicating terms that cancel against each other. For example,

$$
C_{|1| 01}=\left|\oint \frac{d z^{\prime}}{1-z^{\prime}}\right| \int_{[0,1]} \frac{d z}{z} \int_{[0, z]} \frac{d z^{\prime \prime}}{1-z^{\prime \prime}}=\frac{(-2 \pi i)^{1}}{1 !} \mathrm{MZV}_{0,1}
$$

The $\mid$ in the equation above symbolically denotes the dividing of the integral rather than an absolute value.

By analytically continuing the HPL functions of $z$ in the fishnet correlator expansion, this continuation technique provides equivalent results to the $\log (1-z)$ replacement while drastically reducing computation time. 


\section{Froissart-Gribov formula and cancellation of spurious poles}

In section 3.1, we claimed that all the spurious poles cancel against each other in the Sommerfeld-Watson resummation for flat space scattering. In this appendix, we provide justification. To understand the necessary cancellations, we need a concrete expression for the coefficients $a_{J}^{t, u}$ known as the Froissart-Gribov formula. In brief (this is reviewed in $[18,19]$, see also $[22,28]$ ) we may use the orthogonality of spherical harmonics to write partial wave coefficients (for integer $J$ ) as an integral over $[-1,1]$ against the polynomial solution $C_{J}$, which is equal to an integral over the discontinuity of the nonpolynomial

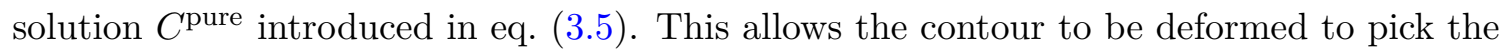
discontinuities of $F(x)$ :

$$
\begin{aligned}
a_{J} & =\frac{\Gamma\left(J+\frac{d}{2}\right)}{\sqrt{\pi} \Gamma\left(\frac{d-1}{2}\right) \Gamma(J+1)} \int_{-1}^{1} d x\left(1-x^{2}\right)^{\frac{d-3}{2}} F(x) \tilde{C}_{J}(x) \\
& =\frac{1}{i \pi} \oint_{[-1,1]} d x\left(\frac{x^{2}-1}{4}\right)^{\frac{d-3}{2}} F(x) C_{2-d-J}^{\text {pure }}(x) \\
\Rightarrow \quad a_{J}^{t} & =\int_{x_{0}}^{\infty}\left(\frac{x^{2}-1}{4}\right)^{\frac{d-3}{2}} C_{2-d-J}^{\text {pure }}(x) \frac{F(x+i 0)-F(x-i 0)}{i \pi}
\end{aligned}
$$

where $\tilde{C}_{J}(x)$ is just the hypergeometric function in eq. (A.5) (without $\Gamma$-factors), the contour on the second line encircles the cut of $C_{2-d-J}^{\text {pure }}(x)$ counter-clockwise, and on the third line we assumed that singularities of $F(x)$ consist of a right-cut for $x>x_{0}$ (and a left-cut for $x<-x_{0}$ which give $a_{J}^{u}$ in the decomposition (3.2)). A technical comment: the Froissart-Gribov integral (C.3) is valid for $J$ large enough that we can ignore arcs at infinity: $\operatorname{Re}(J)>j_{*}$ if $F \sim x^{j *}$. To the left of that, the analytic continuation of $a_{J}^{t}$ need not agree with the coefficients entering eq. (3.1), whence the subtraction terms in eq. (3.3).

We can now explain the two mechanisms responsible for spurious pole cancellation.

We begin with the cancellation of type-2 and type-3 poles defined above eq. (3.7). The Froissart-Gribov integral (C.3) produces singularities for two reasons: "physical" singularities from divergences of the integral, and "spurious" poles due to the integrand itself. It is helpful to denote the two solutions of the Gegenbauer equation, for a given value of $J$, as "small" and "large" depending on whether they vanish or grow as $x \rightarrow \infty$. Large solutions can have poles with residue proportional to the small solution. In the right half-plane the small solution is $C_{2-d-J}^{\text {pure }}$ but the roles get exchanged when $\operatorname{Re}(J)=-\frac{d-2}{2}$, and so it acquires the following spurious left pole:

$$
\underset{J=-\frac{d-2}{2}-m}{\operatorname{Res}} C_{2-d-J}^{\text {pure }}(x) \propto C_{-\frac{d-2}{2}-m}^{\text {pure }}(x) \quad(m=1,2,3 \ldots) .
$$

These are the spurious left-poles of $a_{J}$ called type-2 above eq. (3.7). On the other hand, the right-poles of type-3 come from the combination $\left(P_{J}(x)-C_{J}^{\text {pure }}(x)\right)$ for which we deform the contour to the right in deriving eq. (3.7). Since $P_{J}$ is pole-free on the right, this combination has the same spurious pole as the large solution $-C_{J}^{\text {pure }}(x)$. Thus in the contour deformation argument leading to eq. (3.7) we see that all spurious poles come from 
the following which is always a product of small and large solution:

$$
\frac{e^{-i \pi J} a_{J}^{t}+a_{J}^{u}}{\sin (\pi J)} C_{J}^{\text {pure }}(x) \rightarrow \begin{cases}\text { "large" } \times \text { "small", } & \text { left half-plane }, \\ \text { "small" } \times \text { "large", } & \text { right half-plane, }\end{cases}
$$

and it is easy to see that the residue of both left- and right- spurious poles is the product of the same two small solutions (one hidden in $a_{J}$ and the explicit function of $x$ ), allowing them to precisely cancel. It is interesting to check directly that the proportionality constant in eq. (C.4) is precisely the residue of gamma factors in eq. (3.5), as guaranteed by the general argument just given.

We stress that we are not excluding the possibility that $a_{J}$ may contain a physical pole at $J=-\frac{d-2}{2}-m$ (coming from a large- $x$ divergence in the Froissart-Gribov integral). Rather, the message is that in such a case the "physical" residue to be included in the Regge formula (3.7) is the residue of $a_{J}$ minus a spurious part proportional to $a_{-\frac{d-2}{2}+m}^{t}$ (similar to the way $\Delta$-plane spurious poles are treated in eq. (3.9) of [22]).

The second mechanism, responsible for cancellation of type- 4 poles, is the identity:

$$
a_{J}^{t}+(-1)^{J} a_{J}^{u}=0 \quad(\text { for } J=-1,-2,-3 \ldots)
$$

valid in generic theories which follows from the explicit $1 / \Gamma(J+1)$ in eq. (C.1). By generic we mean that the combination $a_{J} / \sin (\pi J)$ has a pole at negative integer $J$ if only if the Froissart-Gribov formula exhibits a large- $x$ divergence at that exponent, which is nongeneric. We note that these mechanisms are only distinct in generic spacetime dimension: for $\mathrm{SO}(d)$ partial waves with $d$ even, type-3 and type-4 poles occur at the same location. This case is discussed in appendix A of [19]. The overall cancellations (and the result (3.7)) remain valid but one has to use both mechanism at the same time.

\section{Lorentzian inversion of Regge block in $d=2$ and $d=4$}

In this appending we compute the pairing defined in eq. (3.31) between "funny block" and Regge block, which we recall here for convenience:

$$
\frac{\kappa_{\Delta+J}^{(a, b)}}{4} \int_{0}^{1} d z d \bar{z} \mu(z, \bar{z}) G_{J+d-1, \Delta+1-d}^{(-a,-b)}(z, \bar{z}) \frac{R_{\Delta, J}^{(a, b)}(z, \bar{z})}{2 \kappa_{\Delta^{\prime}+J^{\prime}}^{(a, b)}} \equiv P_{\Delta, J ; \Delta^{\prime}, J^{\prime}}^{(a, b)} .
$$

For technical reasons, we were only able to compute this pairing in $d=2$ and $d=4$ spacetime dimensions. Two-dimensional blocks admit the following simple form:

$$
G_{\Delta, J}^{(a, b)}(z, \bar{z})=\frac{k_{\Delta-J}^{(a, b)}(z) k_{\Delta+J}^{(a, b)}(\bar{z})+(z \leftrightarrow \bar{z})}{1+\delta_{J, 0}} \quad(d=2)
$$

where $k_{\Delta-J}^{(a, b)}(z)$ stand for hypergeometric functions defined in eq. (A.2). They are eigenfunctions of the $\mathrm{SL}(2, \mathrm{R})$ Casimir, which we will need shortly:

$$
\left(z^{2} \partial_{z}(1-z) \partial_{z}-(a+b) z^{2} \partial_{z}-a b\right) k_{\beta}^{(a, b)}(z)=\frac{1}{4} \beta(\beta-2) k_{\beta}^{(a, b)}(z) .
$$


From the defining property (3.19) it is easy to see that the Regge block must admit a factorized expression similar to eq. (D.2), with $k$ replaced by involving $k^{\prime}$, which is the solution to same equation but whose discontinuity around $z=1$ vanishes:

$$
\begin{aligned}
k_{\beta}^{\prime(a, b)}(z) & =k_{\beta}^{(a, b)}(z)-\kappa_{2-\beta}^{\prime(a, b)} k_{2-\beta}^{(a, b)}(z) \\
& =\frac{\pi}{\Gamma(1+a+b)} \frac{r_{\beta}^{(-a,-b)}}{s_{\beta}^{(-a,-b)}} z^{\frac{\beta}{2}}{ }_{2} F_{1}\left(\frac{\beta}{2}+a, \frac{\beta}{2}+b, 1+a+b, 1-z\right) .
\end{aligned}
$$

Evaluating the definition (3.17) in $d=2$ we find exactly this!

$$
R_{\Delta, J}^{(a, b)}=k_{\Delta-J}^{\prime(a, b)}(z) k_{2-\Delta-J}^{\prime(a, b)}(\bar{z})+(z \leftrightarrow \bar{z}) .
$$

A similar substitution works for the $d=4$ blocks in eq. (A.1). The pairing then reduces to the following one-dimensional pairing between $k$ and $k^{\prime}$ blocks:

$$
P_{\beta, \beta^{\prime}}^{(a, b) 1 d} \equiv \int_{0}^{1} \frac{d z}{z^{2}} k_{\beta}^{(-a,-b)}(z) k_{\beta^{\prime}}^{(a, b)}(z) .
$$

To compute this, we use the Casimir equation (D.3) and integrate by parts, which gives

$$
\begin{aligned}
& \frac{1}{4}\left(\beta(\beta-2)-\beta^{\prime}\left(\beta^{\prime}-2\right)\right) P^{(a, b) 1 d} \\
& \quad=\lim _{z \rightarrow 1}(1-z)\left[k_{\beta}^{(-a,-b)}(z)\left(\overleftarrow{\partial}_{z}-\vec{\partial}_{z}+\frac{a+b}{1-z}\right) k_{\beta^{\prime}}^{(a, b)}(z)\right] \\
& \quad=\left(1-\beta^{\prime}\right) \frac{r_{\beta}^{(a, b)}}{r_{2-\beta^{\prime}}^{(a, b)}} .
\end{aligned}
$$

The result is simple because only the regular part of $k_{\beta}^{(-a,-b)}(z)$ contributes to the limit. Substituting this into eq. (D.1) for both $d=2$ and $d=4$, and we find a common, compelling expression for both:

$$
\begin{aligned}
P_{\Delta, J ; \Delta^{\prime}, J^{\prime}}^{(a, b)}= & \frac{r_{J+d-\Delta^{\prime}}^{(a, b)} r_{J^{\prime}+\Delta^{\prime}}^{(-a,-b)}}{r_{J^{\prime}+d-\Delta^{\prime}}^{(a, b)} r_{J+\Delta}^{(-a,-b)}} \frac{4(J+\Delta-1)\left(J^{\prime}+d-\Delta^{\prime}-1\right)}{\left(\Delta-\Delta^{\prime}+J-J^{\prime}\right)\left(\Delta-\Delta^{\prime}-J+J^{\prime}\right)\left(\Delta-\tilde{\Delta}^{\prime}-J+\tilde{J}^{\prime}\right)\left(\Delta-\tilde{\Delta}^{\prime}+J-\tilde{J}^{\prime}\right)} \\
& +\left(\Delta^{\prime} \text { shadow }\right) .
\end{aligned}
$$

This formula is further discussed in the main text, see eq. (3.32), where it is interpreted as a Plancherel formula for the $\mathrm{SO}(d, 2)$ group. The common denominator of the two combined lines suggests that the Casimir trick used in $1 \mathrm{~d}$ could be extended to general spacetime dimensions using a eighth order differential operator, but we found this idea somewhat challenging to implement.

Open Access. This article is distributed under the terms of the Creative Commons Attribution License (CC-BY 4.0), which permits any use, distribution and reproduction in any medium, provided the original author(s) and source are credited. 


\section{References}

[1] R.C. Brower, J. Polchinski, M.J. Strassler and C.-I. Tan, The Pomeron and gauge/string duality, JHEP 12 (2007) 005 [hep-th/0603115] [INSPIRE].

[2] L. Cornalba, Eikonal methods in AdS/CFT: Regge theory and multi-reggeon exchange, arXiv:0710.5480 [INSPIRE].

[3] M.S. Costa, V. Goncalves and J. Penedones, Conformal Regge theory, JHEP 12 (2012) 091 [arXiv:1209.4355] [INSPIRE].

[4] J. Maldacena, S.H. Shenker and D. Stanford, A bound on chaos, JHEP 08 (2016) 106 [arXiv: 1503.01409] [INSPIRE].

[5] X.O. Camanho, J.D. Edelstein, J. Maldacena and A. Zhiboedov, Causality constraints on corrections to the graviton three-point coupling, JHEP 02 (2016) 020 [arXiv:1407.5597] [INSPIRE].

[6] T. Hartman, S. Kundu and A. Tajdini, Averaged null energy condition from causality, JHEP 07 (2017) 066 [arXiv: 1610.05308] [INSPIRE].

[7] J. Liu, D. Meltzer, D. Poland and D. Simmons-Duffin, The Lorentzian inversion formula and the spectrum of the 3d O(2) CFT, JHEP 09 (2020) 115 [Erratum ibid. 01 (2021) 206] [arXiv:2007.07914] [INSPIRE].

[8] S. Caron-Huot, Y. Gobeil and Z. Zahraee, The leading trajectory in the $2+1 D$ Ising CFT, arXiv:2007.11647 [INSPIRE].

[9] L. Cornalba, M.S. Costa and J. Penedones, Eikonal approximation in AdS/CFT: resumming the gravitational loop expansion, JHEP 09 (2007) 037 [arXiv:0707.0120] [INSPIRE].

[10] D. Li, D. Meltzer and D. Poland, Conformal bootstrap in the Regge limit, JHEP 12 (2017) 013 [arXiv: 1705.03453] [INSPIRE].

[11] M.S. Costa, T. Hansen and J. Penedones, Bounds for OPE coefficients on the Regge trajectory, JHEP 10 (2017) 197 [arXiv: 1707.07689] [INSPIRE].

[12] P. Kravchuk, J. Qiao and S. Rychkov, Distributions in CFT. Part I. Cross-ratio space, JHEP 05 (2020) 137 [arXiv:2001.08778] [INSPIRE].

[13] O. Gürdoğan and V. Kazakov, New integrable 4D quantum field theories from strongly deformed planar $\mathcal{N}=4$ supersymmetric Yang-Mills theory, Phys. Rev. Lett. 117 (2016) 201602 [Addendum ibid. 117 (2016) 259903] [arXiv:1512.06704] [INSPIRE].

[14] N. Gromov, V. Kazakov and G. Korchemsky, Exact correlation functions in conformal fishnet theory, JHEP 08 (2019) 123 [arXiv: 1808.02688] [INSPIRE].

[15] G.P. Korchemsky, Exact scattering amplitudes in conformal fishnet theory, JHEP 08 (2019) 028 [arXiv: 1812.06997] [INSPIRE].

[16] S. Dutta Chowdhury, P. Haldar and K. Sen, On the Regge limit of fishnet correlators, JHEP 10 (2019) 249 [arXiv: 1908.01123] [InSPIRE].

[17] S.D. Chowdhury, P. Haldar and K. Sen, Regge amplitudes in generalized fishnet and chiral fishnet theories, JHEP 12 (2020) 117 [arXiv: 2008.10201] [INSPIRE].

[18] P.D.B. Collins, An introduction to Regge theory and high-energy physics, Cambridge Monographs on Mathematical Physics, Cambridge University Press, Cambridge U.K. (2009). 
[19] S. Donnachie, H.G. Dosch, O. Nachtmann and P. Landshoff, Pomeron physics and QCD, Cambridge University Press, Cambridge U.K. (2004) [INSPIRE].

[20] D. Simmons-Duffin, D. Stanford and E. Witten, A spacetime derivation of the Lorentzian OPE inversion formula, JHEP 07 (2018) 085 [arXiv: 1711.03816] [INSPIRE].

[21] D. Simmons-Duffin, Projectors, shadows, and conformal blocks, JHEP 04 (2014) 146 [arXiv: 1204.3894] [INSPIRE].

[22] S. Caron-Huot, Analyticity in spin in conformal theories, JHEP 09 (2017) 078 [arXiv: 1703.00278] [INSPIRE].

[23] P. Kravchuk and D. Simmons-Duffin, Light-ray operators in conformal field theory, JHEP 11 (2018) 102 [arXiv: 1805.00098] [InSPIRE].

[24] M. Isachenkov and V. Schomerus, Integrability of conformal blocks. Part I. Calogero-Sutherland scattering theory, JHEP 07 (2018) 180 [arXiv:1711.06609] [INSPIRE].

[25] T.G. Raben and C.-I. Tan, Minkowski conformal blocks and the Regge limit for Sachdev-Ye-Kitaev-like models, Phys. Rev. D 98 (2018) 086009 [arXiv:1801.04208] [INSPIRE].

[26] F. Kos, D. Poland and D. Simmons-Duffin, Bootstrapping the $O(N)$ vector models, JHEP 06 (2014) 091 [arXiv:1307.6856] [INSPIRE].

[27] D. Maître, HPL, a Mathematica implementation of the harmonic polylogarithms, Comput. Phys. Commun. 174 (2006) 222 [hep-ph/0507152] [INSPIRE].

[28] M. Correia, A. Sever and A. Zhiboedov, An analytical toolkit for the S-matrix bootstrap, arXiv:2006.08221 [INSPIRE]. 